\title{
Low Mass Stars and Substellar Objects in the NGC 1333 Molecular Cloud
}

\author{
Bruce A. Wilking ${ }^{1}$ \\ Department of Physics and Astronomy, University of Missouri-St. Louis \\ 8001 Natural Bridge Road, St. Louis, MO 63121 \\ bwilking@umsl.edu \\ Michael R. Meyer \\ Steward Observatory, The University of Arizona, Tucson, AZ 85721 \\ mmeyer@gould.as.arizona.edu \\ Thomas P. Greene ${ }^{1}$ \\ NASA/Ames Research Center, M.S. 245-6 \\ Moffett Field, CA 94035-1000 \\ tgreene@mail.arc.nasa.gov \\ Ayman Mikhail ${ }^{1}$ \\ Department of Physics and Astronomy, University of Missouri-St. Louis \\ 8001 Natural Bridge Road, St. Louis, MO 63121 \\ and \\ Glenn Carlson \\ Department of Physics and Astronomy, University of Missouri-St. Louis \\ 8001 Natural Bridge Road, St. Louis, MO 63121 \\ gcarlson@mail.win.org
}

\begin{abstract}
We present the results of near-infrared imaging and low-resolution nearinfrared spectroscopy of low mass objects in the NGC 1333 molecular cloud. A JHK survey of an $11.4^{\prime} \mathrm{x} 11.7^{\prime}$ area of the northern cluster was conducted to
\end{abstract}

\footnotetext{
${ }^{1}$ Visiting Astronomer at the Infrared Telescope Facility, which is operated by the University of Hawaii under contract from the National Aeronautics and Space Administration.
} 
a sensitivity of $\mathrm{K} \leq 16 \mathrm{mag}$. Using near-infrared magnitudes and colors from this and previously published surveys, twenty-five brown dwarf candidates were selected toward the high extinction cloud core. Spectra in the K band were obtained and comparisons of the depths of water vapor absorption bands in our candidate objects with a grid of dwarf, subgiant, and giant standards were made to derive spectral types. These data were then used to derive effective temperatures and stellar luminosities which, when combined with theoretical tracks and isochrones for pre-main sequence objects, resulted in estimates for their masses and ages. The models suggest a median age for the sample of $<1$ Myr with substellar masses for at least 9 of the candidates including the x-ray flare source ASR 24. Surface gravities have been estimated for the brown dwarf candidates and, for a given spectral type, found to resemble more closely dwarfs than giants. Using the near-infrared imaging data and age estimates from the spectroscopic sample, an extinction-limited sample in the northern cluster was defined. Consistent with recent studies of other young clusters, this sample exhibits an accretion disk frequency of $0.75 \pm 0.20$ and a mass spectrum slope across the hydrogen-burning limit of $\alpha \leq 1.6$ where $\mathrm{dN} / \mathrm{dM} \propto \mathrm{M}^{-\alpha}$.

Subject headings: stars: pre-main-sequence, brown dwarfs - infrared: stars ISM: individual (NGC 1333)

\section{Introduction}

The discovery and characterization of brown dwarfs in young clusters can help answer fundamental questions about the star formation process. Do brown dwarfs dominate in number or mass over low mass stars in young clusters? Do the mass functions of young clusters exhibit a characteristic mass and do they resemble the field star Initial Mass Function (IMF)? Are there differences between the infrared or x-ray properties of young low mass stars and brown dwarfs that would suggest separate formation mechanisms? For example, it has been suggested that some brown dwarfs are ejected from young, multiple star systems thus limiting their accretion phase (Reipurth \& Clarke 2001). Studies of the mass functions of young clusters rely critically on the substellar population to define the peaks of the mass functions and possible low-mass cut-offs (e.g., Hillenbrand \& Carpenter 2000; Luhman et al. 2000; Béjar et al. 2001; Najita et al. 2000; Muench et al. 2002). Such studies will not only help establish whether there are significant variations in the mass functions between young clusters but if these depend on the physical conditions of the cluster gas (Briceno et al. 2002). 
Young brown dwarfs are readily observed in near-infrared surveys of dark clouds since their luminosity is derived from gravitational contraction as well as a brief period of deuterium burning (e.g., Burrows et al. 1997). For example, models predict that in the nearest star-forming regions an object with a mass of $0.04 \mathrm{M}_{\odot}$ will have a $\mathrm{K}$ magnitude of 12.4 at an age of 1 Myr (D'Antona \& Mazzitelli 1998). Recent infrared surveys of young clusters such as the Orion Nebula Cluster (ONC) and IC 348 suggest sizable populations of brown dwarfs (e.g. Hillenbrand \& Carpenter 2000; Luhman et al. 2000; Muench et al. 2002; Muench et al. 2003). However due to an ambiguity in the derived age and mass, mass estimates from infrared photometric data alone are reliable only in a statistical sense. Determination of the effective temperature through spectroscopy removes this ambiguity and leads to more accurate determinations of the visual extinction and stellar luminosity, and with the help of models, the age and mass. Hence, potential substellar objects can be identified and studied on an individual basis. Infrared spectroscopy is ideal for confirming the nature of young brown dwarf candidates because of the obscuration by dust inherent in star-forming regions and the presence of broad water vapor absorption bands in cool photospheres that can be used to derive effective temperatures using low resolution ( $\mathrm{R} \sim 300)$ spectra (e.g., Wilking, Greene, \& Meyer 1999, hereafter WGM).

The NGC 1333 reflection nebula and its associated dark cloud L1450 are part of a chain of molecular clouds in the Perseus region (e.g., Sargent 1979; Loren 1976). Analysis of Hipparcos data suggests a distance of 300 pc (de Zeeuw et al. 1999; Belikov et al. 2002). The observations of emission-line stars and Herbig-Haro objects first established NGC 1333 as an active region of star formation (Herbig \& Rao 1972; Herbig 1974). Surveys of the cloud at near-infrared, far-infrared, and submillimeter wavelengths have revealed that it is forming primarily low mass stars with the most massive young stars, SVS 3 and BD+30 549, exhibiting spectral types in the range of B6-B9 (e.g., Strom, Vrba, \& Strom 1976; Harvey, Wilking, \& Joy 1984; Aspin et al. 1994; Lada, Alves, \& Lada 1996; Jennings et al. 1987; Sandell \& Knee 2001, Aspin 2003). Most of the young stellar objects (YSOs) in the NGC 1333 cloud are concentrated in the highest column density gas and are arranged into a northern and southern cluster, each with about 70 members.

We utilized deep $(\mathrm{K} \leq 16)$ near-infrared surveys of the NGC 1333 cloud core, including our new survey of the northern cluster, to select 25 brown dwarf candidates among the low mass population. Infrared spectra of the candidates were obtained, in addition to a collection of dwarf, subgiant, and giant standards, and a water vapor index was developed to yield estimates for their effective temperatures assuming dwarf to subgiant surface gravities. Using theoretical tracks and isochrones, we used our derived effective temperatures and stellar luminosities to identify brown dwarfs and to investigate their collective properties such as mass, age, infrared excess, x-ray emission, and surface gravity. Finally, we use 
our survey of the northern cluster and the median age of the brown dwarf candidates to assemble an extinction-limited sample of YSOs in the NGC 1333 core. We use this sample to explore the stellar density, disk frequency, and the broad features of the mass spectrum for comparsion with other young clusters and the field star IMF.

\section{Observations and Reduction}

To assist in the identification of brown dwarf candidates, we first obtained a new, deep near-infrared survey of the northern cluster. These data were supplemented with published infrared surveys of the southern cluster to construct a sample of candidates for near-infrared spectroscopy.

\subsection{A New Infrared Survey of the Northern Cluster}

Near-infrared images of the NGC 1333 northern cluster were obtained using the 1.6 meter telescope at Mt. Bigelow (MBO) near Tucson, AZ on the nights of 1999 October 2123. The University of Arizona's $256 \times 256 \mathrm{HgCdTe}$ Infrared Imager was used at wavelengths of $\mathrm{J}(1.25 \mu \mathrm{m}), \mathrm{H}(1.6 \mu \mathrm{m})$, and $\mathrm{K}(2.2 \mu \mathrm{m})$ with a pixel scale of $0.92^{\prime \prime}$ pixel $^{-1}$ (see Rieke et al. 1993 for a description of the instrument).

The northern cluster was mapped using a 5x5 raster pattern with half-frame overlap. The total area covered was $11.4^{\prime} \times 11.7^{\prime}$. The southwest corner of the mosaic is located at $\mathrm{RA}(\mathrm{J} 2000)=3^{h} 28^{m} 40.8^{s}, \mathrm{DEC}(\mathrm{J} 2000)=+31^{\circ} 18^{\prime} 19^{\prime \prime}$ and the northeast corner at RA(J2000) $=3^{h} 29^{m} 41.4^{s}, \operatorname{DEC}(\mathrm{J} 2000)=+31^{\circ} 29^{\prime} 56^{\prime \prime}$. The boundaries of the survey relative to the high column density gas in the core are shown in Fig. 1. Integration times per frame were 30 sec, $20 \mathrm{sec}$, and $10 \mathrm{sec}$ at $\mathrm{J}, \mathrm{H}$, and $\mathrm{K}$, respectively. Individual frames were sky-subtracted using a median-combined image from a subset of frames within the $5 \times 5$ raster that was free of bright stars. The same median-combined image, with dark current subtracted, was used to flat-field frames. Mosaics were constructed for each 5x5 raster using the routine SQMOS within the Infrared Reduction and Analysis Facility (IRAF) ${ }^{2}$. We repeated the $5 \times 5$ raster 10 times at $\mathrm{J}$ and $\mathrm{K}$, and 8 times at $\mathrm{H}$. The mosaics at a given wavelength were combined to yield a total integration time of $20,10.7$, and 6.7 minutes at $\mathrm{J}, \mathrm{H}$, and $\mathrm{K}$, respectively, in

\footnotetext{
${ }^{2}$ IRAF is distributed by the National Optical Astronomy Observatories, which are operated by the Association of Universities for Research in Astronomy, Inc., under cooperative agreement with the National Science Foundation.
} 
the central regions of the field.

Automated source extraction was performed on the final K mosaic using DAOFIND with a threshold of 5 times the rms noise. A total of 213 unsaturated sources were extracted at K. The pixel positions of these sources were used to center apertures for photometry of individual sources in all three bands resulting in a total of 211 sources measured at $\mathrm{H}$ and 187 at J. Aperture photometry was performed using APPHOT in IRAF using an aperture diameter of $3.7^{\prime \prime}$. This aperture was twice the average FWHM of the stellar images and provided the optimum signal-to-noise (Howell 1989). Observations of faint UKIRT standards were used to determine aperture corrections of $0.28,0.29$, and $0.22 \mathrm{mag}$ at $\mathrm{J}, \mathrm{H}$, and $\mathrm{K}$, respectively.

The JHK data are presented in Table 1 in the CIT photometric system. To place our photometry (labeled MBO) in the CIT photometric system, we first compared our magnitudes for 54 sources with $\mathrm{K} \lesssim 13.5 \mathrm{mag}$ with those determined from the SQIID survey of Lada, Alves, \& Lada (1996, hereafter LAL). To place our H and K data in the same photometric system as LAL, only a zero point correction determined from this comparison was needed. The conversion of our J magnitudes used the following correction:

$$
(J-K)_{S Q I I D}=1.03(J-K)_{M B O}-0.14 .
$$

To convert our photometry into the CIT photometric system, which is very close to the SQIID system, we used the prescription of Kenyon et al. (1998). Only the statistical errors in the photometry are quoted in Table 1. However these errors can be exceeded by uncorrected variations in the responsivity across the array due to vignetting that introduce an uncertainty of \pm 0.05 mag. Errors as large as $\pm 0.10 \mathrm{mag}$ can occur in the outer regions of the mosaic due to the lower signal-to-noise. A histogram of the number of sources vs. apparent magnitude was constructed for each wavelength and the turnovers in the histograms were used to estimate the completeness limits of our survey at J, H, and K to be 17.5, 16.5, and 16 mag, respectively. The positions in Table 1 were computed relative to a secondary reference frame defined by Herbig \& Jones (1983) and are accurate to $\pm 0.5^{\prime \prime}$.

\subsection{Selection of Brown Dwarf Candidates}

Brown dwarf candidates were selected based upon their broadband near-infrared colors. The majority of the 25 candidates lie in a region of the $\mathrm{K}$ vs. (H-K) diagram shown in Fig. 2 with $\mathrm{M}<0.1 \mathrm{M}_{\odot}$, and $\mathrm{K}<14$ mag. We adopted an age of $1 \mathrm{Myr}$ based on the $\mathrm{K}$ luminosity function by LAL. The isochrones in Fig. 2 were derived from the models of D'Antona \& Mazzitelli $\left(1994,1997, \mathrm{M}>0.2 \mathrm{M}_{\odot}\right)$ and D'Antona \& Mazzitelli $\left(1998, \mathrm{M} \leq 0.2 \mathrm{M}_{\odot}\right)$ in the

CIT photometric system using intrinsic colors and bolometric corrections for dwarf stars (see 
Appendix A in WGM99). The data and reddening law (Cohen et al. 1981) are also in the CIT photometric system. The list of brown dwarf candidates observed spectroscopically is given in Table 2.

\subsection{Low Resolution Spectroscopy}

Infrared spectroscopic observations were obtained using the 3-m NASA Infrared Telescope Facility at Mauna Kea, Hawaii in 2000 November 10-13. Spectra in the K band were obtained for 25 brown dwarf candidates, eight $\mathrm{M}$ giants, and eight $\mathrm{M}$ subgiants using the 256 x 256 InSb facility infrared camera (NSFCAM) with the HKL grism and a $0.3^{\prime \prime}$ pixel $^{-1}$ scale. The $0.6^{\prime \prime}$ slit allowed us to cover the $2.0 \mu \mathrm{m}-2.5 \mu \mathrm{m}$ band with a resolution of $\mathrm{R}=$ $\lambda / \Delta \lambda \sim 300$. Exposures of the dome interior illuminated with incandescent lamps through the broadband $\mathrm{K}$ filter were used for flat-fielding. A set of observations consisted of the star observed at two positions along the slit, separated by 10 arcsec. A typical exposure time for the brown dwarf candidates was 120 seconds per slit position and limited by variations in the atmospheric $\mathrm{OH}$ emission. Total on-source integration times were typically 2 minutes for the standard star observations and ranged from 8 to 36 minutes for the brown dwarf candidates (see Table 2).

All data were reduced using IRAF. Before extraction, the spectral images in each set were sky-subtracted using their companion image, and divided by a normalized, darksubtracted flat-field. Wavelength calibration was established using $\mathrm{OH}$ emission lines. After extraction, the spectra were median-combined after matching their wavelengths. The resulting spectra were shifted to the same wavelength scale as a a spectrum of either the A0V star HR 1237 or the A1V star HR 1027 that was observed close in time and airmass by cross-correlating telluric absorption lines. Telluric features in the combined spectra were removed by dividing by the appropriate A star spectrum. No attempt was made to restore the true continuum shape of the program objects; to recover the true shape one could multiply each spectrum by a blackbody spectrum corresponding to the effective temperature of the telluric standard. Because of the steep response function of the grism, it was not possible to extrapolate over the $\operatorname{Br} \gamma$ absorption line in the telluric standard and hence we were not sensitive to $\operatorname{Br} \gamma$ emission in the program stars. The signal-to-noise ratios achieved in the normalized continua $\left(\mathrm{SNR}_{\text {cont }}\right)$ of the reduced spectra are listed in the last column of Table 2 . 


\section{Spectroscopic Analysis}

\subsection{Spectral Classification Using a Water Vapor Index}

In the K-band, water vapor absorption bands centered at $1.9 \mu \mathrm{m}$ and $2.5 \mu \mathrm{m}$ provide the best means for deriving spectral types for reddened M stars. These bands are not only sensitive to temperature for cool stars (Jones et al. 1994) but also are well-resolved in low resolution spectra. Water vapor indices have been used to derive spectral types for very low mass YSOs in $\rho$ Ophiuchi (WGM99, Cushing, Tokunaga, \& Kobayashi 2000), IC 348 (Najita, Teide, \& Carr 2000), the ONC (Lucas et al. 2001), the Chamaeleon cloud (Gomez \& Persi 2002), and the Taurus cloud (Itoh, Tamura, \& Tokunaga 2002). In WGM99, we defined a $\mathrm{Q}$ index that was independent of reddening as a measure of the depth of the water vapor absorption:

$$
Q=(F 1 / F 2)(F 3 / F 2)^{1.22}
$$

where F1, F2, and F3 are the average relative fluxes in narrow bands covering $2.07-2.13 \mu \mathrm{m}$, 2.2695-2.2875 $\mu \mathrm{m}$, and 2.40-2.50 $\mu \mathrm{m}$, respectively. The uncertainty in $\mathrm{Q}$ was calculated by using Eqn. 2 and propagating error estimates for the mean fluxes in F1, F2, and F3. The error in $\mathrm{F}_{i}$ is given by $\sigma_{F_{i}}=\mathrm{F}_{i} /\left(\sqrt{N} \times S N R_{\text {cont }}\right)$ where $\mathrm{N}$ is the number of channels in $\mathrm{F}_{i}$ and $S N R_{\text {cont }}$ is the signal-to-noise ratio in the continuum. This assumes the flux uncertainty in each channel of the band $F_{i}$ is the same. As a check, we calculated the uncertainty in the $\mathrm{Q}$ values for 3 brown dwarf candidates by calculating the standard deviation of the ensemble of $\mathrm{Q}$ estimates from individual spectra. The two estimates are in excellent agreement. ${ }^{3}$

A linear fit to a plot of the $\mathrm{Q}$ index vs. the optically-determined $\mathrm{M}$ spectral type for dwarf standards weighted by the uncertainties in $\mathrm{Q}$ for stars from M0.5V-M9V yields the following relation

$$
M V \text { subclass }=(-20.20 \pm 0.30) \times Q+(18.41 \pm 0.35)
$$

with a correlation coefficient of $\mathrm{r}=0.98$ for a sample of 12 measurements. This relation supercedes that in WGM99 as it utilizes an improved wavelength calibration, a redefinition of the F2 band, and a better estimate of the uncertainty in Q. This relation is plotted in blue in Fig. 3.

\footnotetext{
${ }^{3}$ Uncertainties in Q quoted in WGM99 used the sample standard deviations of the average values in the bands F1, F2, F3 and overestimate the true error in Q.
} 


\subsection{The Water Index as a Function of Surface Gravity}

A sample of seven subgiants were selected for infrared spectroscopic observations from the MBM 12 and IC 348 YSO populations (Hearty et al. 2000; Luhman 2001; Luhman et al. 1998; Luhman 1999). The median ages of these clusters are estimated to be $2 \mathrm{Myr}$ and the median ages of the sources in our sample is 1 Myr. As shown in Fig. 3 in green, the linear fit to the subgiant sample is consistent with the dwarf relation and there is no strong sensitivity of the $Q$ index to surface gravities in the dwarf to subgiant range. This is in agreement with the findings of Gorlova et al. (2003) who found the linear relationship between the water index and $M$ spectral type of young cluster objects $(\log (g)=3.68 \pm 0.42)$ closely resembled that of a sample of field stars $(\log (\mathrm{g})=5.17 \pm 0.38)$.

The $\mathrm{Q}$ values for the 8 giants observed show a much greater scatter from a linear fit (shown in red in Fig. 3). In our sample, the giant star spectra are distinct from those of dwarfs and always display weaker water vapor absorption than dwarfs for a given spectral type (see Appendix A). band is not evident until a spectral type of M7III. Yet, the Q index for giants can be smaller than that of dwarfs of the same spectral type due to enhanced absorption by $\mathrm{CO}$ that mimics water vapor absorption in the $2.40-2.50 \mu \mathrm{m}$ band. Variability must also contribute to the scatter in the giant sample; all of the stars from M4-M9 are known variables and two (VY Peg and AW Psc) are Mira variables. We conclude that the Q index is not a useful measure of spectral type for objects with the surface gravities of giant stars.

\section{Properties of the Brown Dwarf Candidates}

\subsection{Spectral Types}

We observed one-half of the 50 sources in an extinction-limited sample defined in Fig. 2 with estimated masses of $\mathrm{M}=0.04-0.10 \mathrm{M}_{\odot}$ and $\mathrm{A}_{v}<10 \mathrm{mag}$. All 25 brown dwarf candidates displayed late-type photospheres with absorption due to water vapor and CO. Spectra are presented in Fig. 4. Since the surface gravities of objects in this sample are likely to resemble closely those of the subgiants observed, spectral types were derived using Eqn. 3 which is appropriate for both subgiants and dwarfs. Q values and the resulting spectral classifications for the brown dwarf candidates are presented in Table 3. Uncertainties in the Q index were $\pm 0.010-0.022$ due to statistical errors alone leading to uncertainties in the $\mathrm{M}$ spectral type of $\pm 0.2- \pm 0.4$ subclasses. The derived spectral types vary between M2.5 and M8.0, with 17 of 25 candidates displaying spectral types between M6.0 and M8.0. Visual extinctions were derived using the relation $\mathrm{A}_{v}=9.09 \times\left[(J-H)_{o b s}-(J-H)_{0}\right]$ where $(J-H)_{0}$ is the instrinsic color for a given spectral type (WGM99). The values for $\mathrm{A}_{v}$ are $\leq 4$ mag for all but 2 sources 
and $\leq 7$ mag for all sources, consistent with their locations in the color-color diagram (Fig. 5).

The majority of the candidates appear to be YSOs of very low mass. The projection of this sample on the high column density core $\left(\mathrm{A}_{v}>10 \mathrm{mag}\right)$, coupled with the relatively low visual extinctions of the candidates, minimizes the chance that some objects are background $\mathrm{M}$ stars. In addition, fifteen of the 25 candidates are known x-ray sources, including 3 tentative detections (Getman et al. 2002, Preibisch 2003). The detection of x-ray emission argues in favor of the youth of this sample, as x-ray luminosity and stellar activity are known to decline with age for K and M stars (Fleming, Schmitt, \& Giampapa 1995). Finally, we estimate the number of foreground stars at this galactic latitude and longitude using star count models. Using the Wainscoat et al. (1992) models, which have been found to agree with the observed star counts from the 2MASS survey to within 15\% (Carpenter 2000), we predict at most 2 foreground objects in the sample of 25.

\subsection{Infrared Excesses}

There are no strong infrared excesses among the spectroscopic sample of brown dwarf candidates. The candidates are plotted on a $(\mathrm{J}-\mathrm{H})$ vs. $(\mathrm{H}-\mathrm{K})$ diagram in Fig. 5 relative to the loci of giants, dwarfs (through M6), and classical T Tauri stars (CTTS, Meyer, Calvet, \& Hillenbrand 1997). Objects that lie to the right of the reddening band for main sequence stars have possible K excesses. ASR 79 lies to the left of the reddening band, a likely result of an associated infrared nebula known optically as $\mathrm{HH}$ 4. In addition, we have computed the excess emission at $\mathrm{K}$, defined as $\mathrm{r}_{k}=\mathrm{F}_{K_{e x}} / \mathrm{F}_{K_{*}}$, for each source where $\mathrm{F}_{K_{e x}}$ is the K-band flux from circumstellar emission and $\mathrm{F}_{K_{*}}$ is the expected stellar flux at $\lambda=2.2 \mu \mathrm{m} .{ }^{4}$ These values are presented in Table 3 . While moderate K-band excesses are indicated for ASR 38 and MBO 79, the majority of objects have colors consistent with no K-band excess. As noted in WGM99, strong veiling by dust can lead one to derive spectral types using the $Q$ index that are too early by $2-3$ subclasses. It is possible that the calculated spectral types for ASR 38 and MBO 79 are too early by 1-2 subclasses due to moderate veiling, but it is expected that the spectral types for the remaining objects are relatively unaffected.

Objects without strong K-band excess emission may also possess circumstellar disks. Disk luminosities are expected to be lower for very low mass stars and substellar objects

\footnotetext{
${ }^{4} \mathrm{r}_{k}$ is calculated by comparing dereddened observed colors to intrinsic colors, i.e., $\mathrm{r}_{k}=\mathrm{F}_{K e x} / \mathrm{F}_{K}=$ $\left[\left(1+\mathrm{r}_{h}\right)\left(10^{\left[(H-K)-(H-K)_{0}-0.065 * A_{v}\right] / 2.5}\right)\right]-1$. Our estimates for $\mathrm{r}_{k}$ are lower limits since, by dereddening to intrinsic dwarf colors, we have assumed $\mathrm{r}_{j}=\mathrm{r}_{h}=0$ (e.g., Meyer et al. 1997).
} 
resulting in cooler inner disks and little or no K-band excess. Such a trend has been observed in the ONC (Hillenbrand et al. 1998). Indeed, L-band surveys have been shown to be more sensitive to the thermal disk emission in low mass objects (Haisch, Lada, \& Lada 2001). A recent L' survey by Liu, Najita, \& Tokunaga (2003) has revealed that about $80 \%$ of the young brown dwarfs in their sample showed evidence for circumstellar disks.

\subsection{Masses and Ages of the Brown Dwarf Candidates}

We followed the procedure outlined in WGM99 to estimate the masses and ages of the brown dwarf candidates. Briefly, the adopted temperature scale for $\mathrm{M}$ dwarfs derived in WGM99 is based on the modified blackbody fits of Jones et al. (1996). This temperature scale overlaps with the scale adopted by Dahn et al. (2002) for M5-L8 dwarfs and is consistent within mutual uncertainties. Effective temperatures for each brown dwarf candidate derived in this manner are given in Table 3 . Their typical uncertainties of $\pm 95 \mathrm{~K}$ or \pm 0.016 dex are due to nearly equal contributions from the errors in the determination of the spectral type and the temperature scale. However, systematic effects could affect the derived effective temperatures. As alluded to earlier, the presence of moderate veiling in 2 objects could lead us to overestimate their effective temperatures by $\sim 150 \mathrm{~K}$. The assumption of dwarf, rather than subgiant, surface gravities could also systematically affect our results. For spectral types of M2 and later, giant star temperatures are warmer than dwarfs by 300-500 K for stars of the same spectral type (for example see Fig. 4 in Itoh et al. 2002). Hence by assuming a dwarf surface gravity, we may be underestimating the effective temperature of an M2 subgiant by $\sim 150 \mathrm{~K}$ and of an M6-M9 subgiant by $\sim 250 \mathrm{~K}$. With these caveats in mind, we adopt the temperatures listed in Table 3 for our brown dwarf candidates assuming a dwarf temperature scale. Values for $\log \left(\mathrm{L}_{b o l} / \mathrm{L}_{\odot}\right)$ were derived from the absolute J magnitude and are presented in Table 3. The formal uncertainties of $\log \left(\mathrm{L}_{b o l} / \mathrm{L}_{\odot}\right)$ are \pm 0.18 dex and were estimated by propagating errors in the bolometric correction, the $\mathrm{J}$ magnitude, the distance modulus, and the extinction at J.

In Fig. 6, we have plotted our 25 brown dwarf candidates on Hertzsprung-Russell diagrams overlaid with the theoretical tracks and isochrones from the models of of D'Antona \& Mazzitelli (1997,1998, hereafter DM), Baraffe et al. (1998, hereafter BCAH), and Burrows et al. $(1997,1998)$. The DM models use opacities from Alexander \& Ferguson (1994), the full spectrum of turbulence convection model of Canuto \& Mazzitelli (1991), and assume mass fractions of helium, metals, and deuterium of $\mathrm{Y}=0.28, \mathrm{Z}=0.02$, and $\mathrm{X}_{D}=2 \times 10^{-5}$. BCAH models have been extended to include objects with masses as low as $0.02 \mathrm{M}_{\odot}$ and assume a mass fraction of helium $\mathrm{Y}=0.275$, solar metallicity, and a general mixing length parameter 
of $\alpha=1$. The Burrows et al. models are constructed specifically for giant planets and brown dwarfs and assume mass fractions of helium, metals, and deuterium of $\mathrm{Y}=0.25, \mathrm{Z}=0.02$, and $\mathrm{X}_{D}=2 \times 10^{-5}$. As found in most young clusters, there is a scatter in age due in part to real age differences between sources and to uncertainties in luminosities that arise from variations in distance and errors introduced by the photometry and dereddening (the latter are represented by the error bar in Fig. 6). Ages can be overestimated due to underestimating the luminosity of a source or overestimating its temperature. The former could be due to underestimating the extinction toward the source owing to unresolved scattered light (e.g., ASR 79) and the latter could be due to an infrared excess as discussed in Sec. 4.2 (e.g., MBO 79).

For the DM models (Fig. 6a), the brown dwarf candidates have masses ranging from $<0.02 \mathrm{M}_{\odot}$ to $0.25 \mathrm{M}_{\odot}$, with 16 objects at or below the hydrogen-burning limit. The probable brown dwarfs are indicated in the last column of Table 3. The median age for the sample implied by the models is 0.3 Myr. Similar ranges in ages and masses are implied by considering the Baraffe et al models (Fig. 6b). The Burrows et al. models generally yield lower masses and younger ages with 23 objects at or below the hydrogen-burning limit. The median age for the sample implied by these models is $<0.3$ Myr.

\subsection{Surface Gravity Estimates}

Surface gravities $\left(\mathrm{g}=\mathrm{GM} / \mathrm{R}^{2}\right)$ have been estimated for the brown dwarf candidates using the DM mass estimates and the radii derived from the luminosity and effective temperature. Values range from $\log (\mathrm{g})=3.0$ to 4.6 in cgs units with a median value of 3.3. These estimates assume a temperature scale, intrinsic colors, and bolometric corrections derived from dwarf standards. For comparison, the $\log (\mathrm{g})$ of the Sun is 4.4 (Livingston 1999). Surface gravity estimates increase under the assumption of giant properties, primarily due to their higher effective temperatures for a given $M$ spectral type, implying that our estimates of $\log (\mathrm{g})$ are lower limits. Thus, as shown in Fig. 7, the surface gravities of our objects more closely resemble dwarfs than giants, implying that our use of a dwarf temperature scale has led to an underestimation of the temperature for M6-M9 stars of no more than $\sim 250 \mathrm{~K}$.

The dominant source of uncertainty in our mass estimates is the surface gravity dependence of our temperature determinations. The arrows in Fig. 6 indicate the maximum shift in temperature for an object of a given spectral type if its temperature is midway between that of a dwarf and giant rather than that of a dwarf as assumed. While as many as 16 of our candidates have masses at or below the hydrogen burning limit (in the DM models), only 9 are cool enough to be classified as brown dwarfs after undergoing this shift. In Table 
3 we distinguish between objects that lie $250 \mathrm{~K}$ or more to the right of the hydrogen-burning limit ("bd" in last column) and those to the right of the limit but within $250 \mathrm{~K}$ ("bd?"). In the group of nine brown dwarfs is the moderately-veiled source ASR 38, and 4 x-ray sources including the x-ray flare source ASR 24.

\section{Properties of the Northern Cluster}

To explore further the properties of the young stellar and substellar population in NGC 1333, we have defined a subsample of 141 sources from our JHK survey of the northern cluster that lie within a $79 \mathrm{arcmin}^{2}$ box enclosing the highest column density gas. The southwest corner of the box is located at $\mathrm{RA}(\mathrm{J} 2000)=3^{h} 28^{m} 53^{s}, \operatorname{DEC}(\mathrm{J} 2000)=+31^{\circ} 18^{\prime}$ $19^{\prime \prime}$ and the northeast corner at $\mathrm{RA}(\mathrm{J} 2000)=3^{h} 29^{m} 30^{s}$, DEC $(\mathrm{J} 2000)=+31^{\circ} 28^{\prime} 19^{\prime \prime}$. The average visual extinction (as implied by the $\mathrm{C}^{18} \mathrm{O}$ column densities) is greater than 10 mag over $85 \%$ of the area. The total mass of gas from these data is estimated to be $100 \mathrm{M}_{\odot}$, with a factor of 2 uncertainty (Hatchell et al. 2003).

\subsection{Membership and Stellar Density}

The high column density gas in this region should minimize the contamination of the sample by background field stars. To estimate the degree of contamination, we used the $\mathrm{C}^{18} \mathrm{O}$ column densities to subdivide the cloud core into 6 extinction regions as defined by the contours in Fig. 1 with average visual extinctions of 6.5, 13.5, 20.5, 27.5, 34.5, and 41.5 mag. Galactic star count models (Wainscoat et al. 1992) were then used with the corresponding extinction screen to predict the number of foreground and background stars in the field. We find that of the 141 infrared sources in this region, it is estimated no more than 15 are field stars. For an effective radius of $0.44 \mathrm{pc}\left(R_{e f f}=\sqrt{A / \pi}\right.$ where $\mathrm{A}$ is the area of the box $)$, this

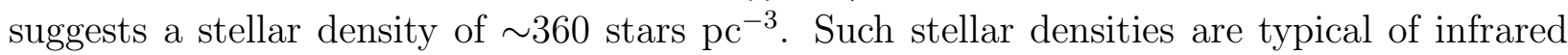
clusters and underscore their stability against tidal disruption by nearby clouds while they are gravitationally bound by the molecular gas (e.g., Wilking 2001).

\subsection{An Extinction-Limited Sample}

Using the median age derived for the spectroscopic sample, we will define an unbiassed sample of YSOs to explore the disk frequency and mass function of the northern cluster. First, estimates for the extinction toward each member of subsample are needed. Depending 
on their location in the $(\mathrm{J}-\mathrm{H})$ vs. $(\mathrm{H}-\mathrm{K})$ color-color diagram, and the $\mathrm{M}_{J}$ vs. $(\mathrm{J}-\mathrm{H})$ colormagnitude diagram, objects were dereddened either to the CTTS locus (color-color diagram) or to a pre-main sequence isochrone (color-magnitude diagram). If the (J-H) vs. (H-K) colors of objects in our sample lie within the reddened CTTS locus (Fig. 5), and the dereddened absolute $\mathrm{J}$-band magnitude is consistent with a mass greater than $0.1 \mathrm{M}_{\odot}$, the source was dereddened to the CTTS locus. The CTTS locus is only valid for stellar mass objects with spectral types earlier than M6 which corresponds roughly to the temperature of an object near the hydrogen-burning limit for the age of the cluster. For objects that lie within the reddened main sequence in the color-color diagram, as well as those within the CTTS locus whose dereddened $\mathrm{M}_{J}$ suggest a mass below $0.1 \mathrm{M}_{\odot}$, the reddening was estimated by projecting objects in the color-magnitude diagram to an isochrone consistent with the cluster age derived above from the $\mathrm{H}-\mathrm{R}$ diagram.

Once the extinction has been estimated, then the absolute J-band magnitude can be calculated. Using intrinsic colors and bolometric corrections for very late-type dwarf stars (see Appendix A in WGM99), we transformed the DM models to estimate the mass from the absolute $J$ magnitude. In order to define a complete sample unbiassed toward stars of higher mass, we defined an extinction-limited sample over a fixed mass range. Adopting an age of 0.3 Myr for the low mass cluster members as suggested by the spectroscopic observations, we sampled 46 objects down to $0.04 \mathrm{M}_{\odot}$ with $\mathrm{A}_{V}<13^{m}$. This is the minimum mass for which we can reliably estimate an age from our spectroscopic survey. Of these, two objects exhibit extreme infrared colors similar to flat-spectrum and Class I sources in the Taurus dark cloud (cf. Kenyon and Hartmann, 1995). These objects cannot be dereddened and are removed from the sample. Of the remaining 44, five exhibit colors similar to early-type (B-A-F) YSOs (e.g. Hillenbrand et al. 1992; Lada and Adams, 1992), 12 are stellar mass objects within the reddened main sequence, 11 are stellar mass objects within the reddened CTTS locus, and 16 are brown dwarf candidates.

The total mass of stellar and substellar objects in this sample is about $8 \mathrm{M}_{\odot}$. For the 7 objects in common between the extinction-limited sample and the spectroscopic sample, mass estimates for individual objects agree to within a factor of 2 which is consistent with previous applications of this technique (Meyer et al. 2000). It is important to note, however, that the mean mass of the two samples is virtually identical (within 10\%) emphasizing that the assumption of a common age yields a meaningful mass estimate for a statistically significant ensemble. The mass of gas associated this region where $\mathrm{A}_{V}<13^{m}$ is estimated to be $\sim 70 \mathrm{M}_{\odot}$. Given the total mass of young stars, the efficiency of star formation would be $10 \%$, with a factor of 2 uncertainty from the mass estimate alone. This value is consistent with that found in other young clusters and suggests the eventual dispersal of the cluster once the gas is removed (Carpenter 2000; Lada \& Lada 2003). 


\subsubsection{Disk Frequency}

The fraction of objects exhibiting IR excesses consistent with the presence of active accretion disks within $0.1 \mathrm{AU}$ of the star can be determined from an object's location in the color-color diagram (Fig. 5). Within the $\mathrm{A}_{V}$-limited sample of stellar mass objects defined here $\left(>0.1 \mathrm{M}_{\odot}\right)$, the fraction of YSOs displaying an IR excess is $F_{I R X}=0.57 \pm 0.13$ adopting Poisson errors. Because some T Tauri stars have large inner holes in their disks that removes hot dust associated with JHK excess emission, this inner disk fraction can underestimate the fraction of stars with active accretion disks. Again, using the Taurus dark cloud as a guide (Meyer et al. 1997), we estimate the corrected fraction of active accretion disks at $F_{a c c}=0.75 \pm .20$. These estimates are in excellent agreement with those determined for the entire NGC 1333 cluster from the SQIID survey by LAL and consistent with disk frequencies estimated for clusters of comparable age (e.g., Haisch et al. 2001). We are not able to assess the disk frequency for the substellar sample using the JHK colorcolor diagram alone because the intrinsic colors of late type stars overlap those expected for active accretion disks. Complete spectroscopic samples or longer wavelength photometry are required to assess the disk frequency of ensemble of the substellar objects in NGC 1333.

\subsubsection{Mass Spectrum}

While our sample is not large enough to examine the detailed shape of the cluster's mass spectrum, we can explore its general properties. Among stellar objects, we can calculate the ratio of intermediate mass $\left(1-10 \mathrm{M}_{\odot}\right)$ to low mass $\left(0.1-1 \mathrm{M}_{\odot}\right)$ stars. The ratio $\mathcal{R}=0.08 \pm 0.04$ with the error computed assuming Poisson statistics. This ratio is consistent with being drawn from the field star IMF (e.g., Miller \& Scalo 1979; Kroupa, Tout, \& Gilmore 1993) and is similar to $\mathcal{R}$ values computed from other clusters such as the ONC, Mon R2, Rho Ophiuchi, and IC 348 (Meyer et al. 2000).

Turning to the subset of cluster members whose $\mathrm{M}_{J}$ suggests a mass below $0.1 \mathrm{M}_{\odot}$, we determine the ratio of stellar $\left(0.1-1 \mathrm{M}_{\odot}\right)$ to substellar mass $\left(0.04-0.1 \mathrm{M}_{\odot}\right)$ objects within our extinction-limited sample. The ratio $\mathcal{R}_{s s}=1.1+0.8 /-0.4$ which is a lower limit given possible contamination of the substellar sample by field stars (Sec. 5.1). Hence our value of $\mathcal{R}_{s s}$ sets an upper limit to the slope for the low mass end of the mass spectrum of $\alpha \leq 1.6$ where $\mathrm{dN} / \mathrm{dM} \propto \mathrm{M}^{-\alpha}$. This suggests that the mass of the population in the mass range of 0.04-1 $\mathrm{M}_{\odot}$ is dominated by low mass stars and not brown dwarfs. This upper limit is consistent with values of $\alpha=1-2$ derived from the observed distribution of $\mathrm{L}$ dwarfs in the solar neighborhood (Reid et al. 1999). Our upper limit is also consistent with the slopes derived for other young clusters; these estimates range from $\alpha=0.43$ for the ONC 
(Hillenbrand \& Carpenter 2000) and $\alpha=0.5$ for IC 348 and $\rho$ Oph (Najita et al. 2000; Luhman \& Rieke 1999) to $\alpha=0.8$ to 1.2 for $\sigma$ Orionis cluster (Béjar et al. 2001; Tej et al. 2002).

\subsubsection{Sensitivity to Age}

If we instead take an age of $1 \mathrm{Myr}$ for the cluster associated with NGC 1333, perhaps more appropriate for the mass range $0.1-2.5 \mathrm{M}_{\odot}$ (Aspin 2003; Luhman, priv. comm.), our results do not change significantly. A trend of increasing age with stellar mass is observed toward many young clusters (e.g., Hillenbrand 1997) and it is suspected that this is a problem with the isochrones rather than evidence that the star formation rate varies with age and mass (Palla \& Stahler 1999). For an age of 1 Myr, our extinction-limited sample becomes 52 objects with $\mathrm{A}_{v}<10^{m}$. Of these, two have extreme colors similar to protostars and cannot be dereddened as described above. Of the 50 remaining objects in the sample, five have IR excesses similar to "early-type" stars, 14 are within the stellar reddened main sequence, 11 lie within the CTTS locus, and 20 are brown dwarf candidates. The IR excess fraction is virtually unchanged with $F_{I R X}=0.59 \pm 0.15$ and with a corrected active accretion disk fraction of $F_{I R X}=0.80 \pm 0.20$. The ratio of intermediate to low mass stars is $\mathcal{R}=0.18 \pm 0.14$ which is also consistent with being drawn from the field star IMF. This difference, although formally in agreement with the results obtained assuming an age of $0.3 \mathrm{Myr}$, is to be expected given the luminosity evolution of pre-main-sequence stars over time. Finally, the ratio of stellar to substellar mass objects is $\mathcal{R}_{s s}=0.6 \pm 0.2$ which implies an upper limit to the slope for the mass spectrum at low masses of $\alpha \leq 2$. While formally consistent with the results quoted above, this highlights the sensitivity of our estimate of the substellar IMF with age.

\section{Summary}

We have conducted a new near-infrared survey of the northern cluster embedded in the NGC 1333 cloud. When combined with previous surveys, near-infrared magnitudes and colors were used to select 25 brown dwarf candidates in the high-extinction cloud core. Low resolution infrared spectroscopy in the $\mathrm{K}$ band revealed cool photospheres for all of the candidates. Spectral classifications were achieved using a water vapor index that is independent of reddening and insensitive to surface gravity in the range expected for subgiants and dwarfs. The derived spectral types varied between M2.3 and M8.2, with 17 of 25 candidates displaying spectral types between M6.0 and M8.2. Based upon their superposition on the high extinction cloud core, their high incidence of x-ray activity, and the low number of 
foreground objects predicted by star count models, it is expected that at least $90 \%$ of the sample are very low mass members of the NGC 1333 cluster.

Once spectral types and effective temperatures were determined, stellar luminosities were estimated. Comparisons of these properties with three sets of theoretical models suggest a median age for the sample of $<1 \mathrm{Myr}$ and a substellar mass for at least 9 of the candidates including the x-ray flare source ASR 24. Another 7 candidates lie near the hydrogen-burning limit and are possible brown dwarfs. The largest uncertainty in our mass estimates arises from the effective temperature and the sensitivity of the temperature scale to surface gravity. We have shown that surface gravities estimated for the brown dwarf candidates more closely resemble dwarfs than giants for a given spectral type and hence a dwarf temperature scale is more appropriate than a giant temperature scale for young objects.

Finally, we have used our near-infrared survey to define an extinction-limited sample of YSOs in the high-extinction core to investigate the properties of the northern cluster. For this sample of 44 objects, the the fraction of objects exhibiting IR excesses is $F_{I R X}=$ $0.57 \pm 0.13$, in excellent agreement with previous estimates for this cluster and other clusters of comparable age. Using masses estimated from intrinsic colors and absolute J magnitudes for very late-type dwarf stars and the 0.3 Myr DM models, we find a mass spectrum for intermediate and low mass stars that is indistinguishable from that in other young clusters and the field star IMF. Due to the possible presence of background stars in the sample, only an upper limit to the slope of the mass spectrum at very low and substellar masses can be estimated. The slope of $\alpha \leq 1.6$ is consistent with that found for substellar objects in other young clusters and in the field.

We would like to thank Marcia Rieke, George Rieke, and Erick Young for their assistance with the infrared survey at Mt. Bigelow Observatory. Marcel Bechtoldt, Tony Denault, and Alan Tokunaga are gratefully acknowledged for their efforts in establishing the Internet2 connection from St. Louis to Mauna Kea for the IRTF observations. We would also like to thank Gary Fuller, Jennifer Hatchell, Charles Lada, Kevin Luhman, and Konstantin Getman for their generosity in sharing data in advance of publication. We are grateful to Mike Merrill for his assistance with SQMOS and to Margaret Meixner and Rich Schuler for their efforts with a preliminary NGC 1333 survey using NIRIM at Mt. Laguna Observatory. We benefited from many discussions with John Carpenter, Nadja Gorlova, and Lynne Hillenbrand. BW and AM gratefully acknowledge support from RUI Grant NSF AST 98-20898 and the NASA/Missouri Space Grant Consortium and GC from the Missouri Research Board. 


\section{A. Spectra of M Giants}

Grism spectra were obtained for eight objects of low surface gravity and are presented in Fig. 8. Owing to the high infrared brightness of these objects, the SNRs in the unsmoothed spectra are greater than 100. The giant sample includes the following objects and their optically-determined spectral types: HD 236879 and BD+30 3647 (M0, Jaschek et al. 1978), BD+29 3674 and BD+06 4112 (M2, Jaschek et al. 1978), HD 167654 (M4, Houk \& Swift 1999), BD+27 3478 (M5, Jaschek et al. 1978), VY Peg (M7, Kirkpatrick et al. 1997), CTI+191258.9+280353 (M7, Kirkpatrick et al. 1994), and AW Psc (M9, Solf 1978). We note that the luminosity class for AW Psc is not well-determined.

Features in the giant spectra are distinct from those in the cluster member or dwarf star spectra. The giant star spectra are dominated by the $\mathrm{CO}$ bandheads and display weak $\mathrm{Na} \mathrm{I}$ and Ca I absorption lines. In addition to the four ${ }^{12} \mathrm{C}^{16} \mathrm{O}(\delta \nu=2)$ bandheads normally seen in infrared spectra, also seen is the $(6,4)$ bandhead at $2.414 \mu \mathrm{m}$, the $(7,5)$ bandhead at $2.446 \mu \mathrm{m}$, and perhaps even the $(8,6)$ bandhead at $2.479 \mu \mathrm{m}$ (see Goorvitch 1994 for line list). This leads to an overall depression of the continuum in the $2.4-2.5 \mu \mathrm{m}$ region and hence a Q index such as defined in Eqn. 2 cannot be used to measure the depths of the water bands. Water vapor absorption at $1.9 \mu \mathrm{m}$ is first evident in the spectrum of the M7 III VY Peg but is weak or absent in the M7 III VY Peg CTI+191258.9+280353. This is no doubt related to the pulsation of these variable stars which leads to fluctuations in their effective temperatures.

\section{REFERENCES}

Alexander, D. R. \& Ferguson, J. W. 1994, ApJ, 437, 879

Aspin, C. 2003, AJ, 125, 1480

Aspin, C., Sandell, G., \& Russell, A. 1994, A\&A, 106, 165

Baraffe, I., Charbrier, G., Allard, F, \& Hauschuldt, P. H. 1998, A\&A, 337, 403 (BCAH)

Béjar, V., Martin, E., Zapatero Osorio, M., Rebolo, R., Barrado y Navascués, D., BailerJones, C., Mundt, R., Baraffe, I., Chabrier, C., \& Allard, F. 2001, ApJ, 556, 830

Belikov, A., Kharchenko, N., Piskunov, A., Schilbach, E., \& Scholz, R.-D. 2002, A\&A, 387, 117

Bessell, M. S. \& Brett, J. M 1988, PASP, 100, 1134

Briceno, C., Luhman, K., Hartmann, L., Stauffer, J., \& Kirkpatrick, J. 2002, ApJ, 580, 317 
Burrows, A., Marley, M., Hubbard, W. B., Lunine, J. L., Guillot, T., Saumon, D., Freedman, R., Sudarsky, D., \& Sharp, C. 1997, ApJ, 491, 856

Burrows, A., Sudarsky, D., Sharp, C., Marley, M., Hubbard, W. B., Lunine, J. L., Guillot, T., Saumon, D., Freedman, R. 1998, in Brown Dwarfs and Extrasolar Planets, ASP Conf. Series No. 134, ed. R, Rebolo, E. L. Martin, \& M. R. Zapatero-Osorio, (ASP: San Francisco), p. 354

Canuto, V. M. \& Mazzitelli, I. 1991, ApJ, 370, 295

Carpenter, J. M. 2000, AJ, 120, 3139

Casali, M. \& Harwarden, T. 1992, The JCMT-UKIRT Newsletter, No. 4, 33

Cohen, J. G., Frogel, J. A., Persson, S. E., \& Elias, J. H. 1981, ApJ, 249, 481

Cushing, M., Tokunaga, A., \& Kobayashi, N. 2000, AJ, 119, 3019

Dahn, C. C. et al. 2002, AJ, 124, 1170

D’Antona, F. \& Mazzitelli, I. 1994, ApJS, 90, 467

D'Antona, F. \& Mazzitelli, I. 1997, in Cool Stars in Clusters and Associations, eds. R. Pallavicini \& G. Micela, Mem. S. A. It., 68, n.4. (DM)

D’Antona, F. \& Mazzitelli, I. 1998, priv. comm. (DM)

de Zeeuw, P. T., Hoogerwerf, R., \& de Bruijne, J. H. J., 1999, AJ, 117, 354

Drilling, J. \& Landolt, A. 1999, in Astrophysical Quantities (4th Ed.), ed. A. Cox, (AIP Press: New York), pp. 381-396

Fleming, T., Schmitt, J., \& Giampapa, M. 1995, ApJ, 450, 401

Getman, K., Feigelson, E., Townsley, L., Bally, J., Lada, C., \& Reipurth, B. 2002, ApJ, 575, 354

Gomez, M. \& Persi, P. 2002, A\&A, 389, 494

Goorvitch, D. 1994, ApJS, 95, 535

Gorlova, N. I., Meyer, M. R., Rieke, G. H., \& Liebert, J. 2003, /apj, 593, 1074

Haisch, K. E., Lada, E. A., \& Lada, C. J. 2001, ApJ, 553, L153

Harris, D. L., Morgan, W. W., \& Roman, N.G. 1954, ApJ, 119, 622

Harvey, P. M., Wilking, B. A., \& Joy, M. 1984, ApJ, 278, 156

Hatchell, J., Fuller, G., Richer, J. \& Qualtrough, C. 2003, in preparation

Hearty, T., Neuhauser, R., Stelzer, B., Fernandez, M., Alcala, J., Covino, E., \& Hambaryan, V. 2000, A\&A, 353, 1044 
Herbig, G. H. 1974, Lick Obs. Bull. No. 658

Herbig, G. H. \& Jones, B. F. 1983, AJ, 88, 1040

Herbig, G. \& Rao, N. 1972, ApJ, 174, 401

Hillenbrand, L. 1997, AJ, 113, 1733

Hillenbrand, L. \& Carpenter, J. 2000, ApJ, 540, 236

Hillenbrand, L., Strom, S., Calvet, N., Merrill, M., Gatley, I., Makidon, R., Meyer, M., \& Skrutskie, M. 1998, AJ, 116, 1816

Houk, N. \& Swift, C. 1999, Michigan catalogue of two-dimensional spectral types for the HD stars; Vol. 5 (Ann Arbor, Michigan: Dept. of Astronomy, University of Michigan)

Howell, S. B. 1989, PASP, 101, 612

Itoh, Y., Tamura, M., \& Tokunaga, A. 2002, PASJ, in press

Jaschek, M. 1978, Bull. Inf. Centre Donnees Stellaires, 15, 121

Jennings, R., Cameron, D., Cudlip, W., \& Hirst, C. 1987, MNRAS, 226, 461

Jones, H. R. A., Longmore, A. J., Jameson, R. F., \& Mountain, C. M. 1994, MNRAS, 267, 413

Jones, H. R. A., Longmore, A. J., Allard, F., \& Hauschildt, P. H. 1996, MNRAS, 280, 77

Kenyon, S., Lada, E., \& Barsony, M. 1998, AJ, 115, 252

Kirkpatrick, J. D., Henry, T., \& Irwin, M. J. 1997, AJ, 113, 1421

Kirkpatrick, J. D., McGraw, J. T., Hess, T. R., Liebert, J., \& McCarthy, D. W. 1994, ApJS, 94,749

Kroupa, P., Tout, C. A., \& Gilmore, G. 1993, MNRAS, 262, 545

Lada, C., Alves, J., \& Lada, E. 1996, AJ, 111, 1964 (LAL)

Lada, C. \& Lada, E. 2003, ARA\&A, 41, 57

Liu, M., Najita, J., \& Tokunaga, A. 2003, ApJ, 585, 372

Livingston, W. C. 1999, in Astrophysical Quantities (4th Ed.), ed. A. Cox, (AIP Press: New York), p. 339

Loren , R. B. 1976, ApJ, 209, 466

Lucas, P. W., Roche, P. F., Allard, F. \& Hauschildt, P. H. 2001, MNRAS, 326, 695

Luhman, K. \& Rieke, G. 1999, ApJ, 525, 440

Luhman, K., Rieke, G., Lada, C., \& Lada, E. 1998, ApJ, 508, 347 
Luhman, K., 1999, ApJ, 525, 466

Luhman, K., Rieke, G., Young, E., Cotera, A., Chen, H., Rieke, M., Schneider, G., \& Thompson, R. 2000, ApJ, 540, 1016

Luhman, K. 2001, ApJ, 560, 287

Meyer, M. R., Adams, F. C., Hillenbrand, L. A., Carpenter, J. M., \& Larson, R. B. 2000, in Protostars and Planets IV, eds. V. Mannings, A. P. Boss, \& S. S. Russell, (Arizona Press: Tucson), p. 121

Meyer, M. R., Calvet, N., \& Hillenbrand, L. A. 1997, AJ, 114, 288

Miller, G. E. \& Scalo, J. M. 1979, ApJS, 41, 513

Muench, A., Lada, E., Lada, C., \& Alves, J. 2002, ApJ, 573, 366

Muench, A., Lada, E., Lada, C., Elston, R., Alves, J., Horrobin, M., Levine, J., Raines, N., \& Roman-Zuniga, C. 2003, AJ, 125, 2029

Najita, J. R., Tiede, G. P., \& Carr, J. S. 2000, ApJ, 541, 977

Palla, F. \& Stahler, S. W. 1999, ApJ, 525, 772

Preibisch, T. 2003, A\&A, 401, 543

Reid, I. N., Kirkpatrick, J. D., Liebert, J., Burrows, A., Gizis, J. E., Burgasser, A., Dahn, C. C., Monet, D., Cutri, R., Beichman, C. A., \& Skrutskie, M. 1999. ApJ, 521, 613

Reipurth, B. \& Clarke, C. 2001, AJ, 122, 432

Rieke, M. J., Rieke, G. H., Green, E. M., Montgomery, E. F., \& Thompson, C. L. 1993, Proc. SPIE, 1946, 214

Sandell, G. \& Knee, L. 2001, ApJ, 546, L49

Sargent, A. 1979, ApJ, 233, 163

Solf, J. 1978, A\&AS, 34, 409

Strom, S., Vrba, F., \& Strom, K. 1976, AJ, 81, 314

Tej, A., Sahu, K., Chandrasekar, T., \& Ashok, N. 2002, ApJ, 578, 523

Wainscoat, R. J., Cohen, M., Volk, K., Walker, H., \& Schwartz, D. 1992, ApJS, 83, 111

Wilking, B. A. 2001, in From Darkness to Light, ASP Conference Series Vol. 243, eds. T. Montmerle \& Ph. André, (ASP: San Francisco), p. 353

Wilking, B., Greene, T., \& Meyer, M. 1999, AJ, 117, 469 (WGM99) 


\section{Figure Captions}

Fig. 1. - The distribution of the 25 brown dwarf candidates relative to the high column density gas in the NGC 1333 core. The boxes outline the extents of deep infrared surveys of the northern cluster (this group) and the southern cluster (Aspin et al. 1994). Coordinates are in J2000.0. Contours correspond to the integrated intensity of $\mathrm{C}^{18}(1-0)$ emission in units of $\mathrm{T}_{M B}$ beginning at $2 \mathrm{~K} \mathrm{~km} \mathrm{~s}^{-1}$ and increasing in steps of $0.7 \mathrm{~K} \mathrm{~km} \mathrm{~s}^{-1}$. The contours correspond roughly to $\mathrm{A}_{v}$ values of $10,17,24,31,38,45$, and 52 mag. The $\mathrm{C}^{18}(1-0)$ data were obtained at FCRAO and smoothed to a resolution of 1 arcmin (Hatchell et al. 2003, in preparation).

Fig. 2.- A color-magnitude diagram for the NGC 1333 double cluster. Photometry is taken from this study (northern cluster) or from the survey of Aspin et al. (1994) (southern cluster), transformed into the CIT system. The Aspin et al. (1994) JHK photometry for the southern cluster was transformed into the CIT system using the formulation of Casali \& Harwarden (1992). Isochrones are shown for $1 \mathrm{Myr}\left(0.02-2.0 \mathrm{M}_{\odot}\right)$ and for $5 \times 10^{8}$ years (0.1-2.0 $\mathrm{M}_{\odot}$, labeled "ZAMS"). Reddening lines from the $1 \mathrm{Myr}$ isochrone are shown for selected masses by dashed lines and were calculated using the extinction law derived by Cohen et al. (1981). For comparison, a reddening vector for an $\mathrm{A}_{v}=10$ mag is also shown.

Fig. 3.- The Q index vs. M spectral type for dwarf standards (blue), subgiants (green), and giants (red). Linear fits to the dwarf, subgiant, and giant data are shown in the same color scheme. The dwarf sample is from WGM99. The subgiants in MBM 12 and their

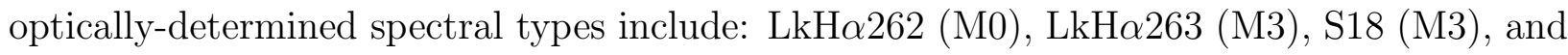
RXJ0258 (M4.5). The subgiants in IC 348 and their optically-determined spectral types include: Luhman source IDs 158 (M5), 312 (M6), and 355 (M8). The giant sample is described in Appendix A.

Fig. 4. - Infrared spectra for the 25 brown dwarf candidates observed with the IRTF grism. Spectra have been smoothed to a resolution of $\mathrm{R}=170$ for display purposes. The removal of telluric absorption lines, accomplished by dividing a spectrum by that of an AV star, leaves a false emission line at the wavelength of $\mathrm{B} \gamma$ which has been blanked out. The wavelengths of absorption lines of $\mathrm{Na}$, $\mathrm{Ca}$, and $6 \mathrm{CO}$ bandheads are marked at the bottom of the plot. The three wavelength ranges averaged to compute the water vapor index $\mathrm{Q}$ are labeled at the top of the plot as F1, F2, and F3. Fig. 4a shows spectra for the sources with spectral types ranging from M2.3 (top) to M5.6 (bottom) as indicated by the increasing depth of the water vapor bands. Fig. 4b shows spectra for the sources ranging from M6.2 to M7.4 and Fig. 4c for sources ranging from M7.4 to M8.2. 
Fig. 5.- A $(J-H) v s .(H-K)$ diagram for the brown dwarf candidates. Photometry is from this study, the study of Aspin et al. (1994), or from Lada et al. (1996) transformed into the CIT photometric system. The loci for the intrinsic colors of dwarfs and giants were adopted from Bessell \& Brett (1998), using their conversion to the CIT system. The loci for classical T Tauri stars is adopted from Meyer et al. (1997). Object symbols for identified $\mathrm{x}$-ray emitters are filled with an "X". An error bar of \pm 0.07 mag is shown that would arise in the colors from uncorrected variations in the array responsivity due to vignetting.

Fig. 6.- Hertzsprung-Russell diagrams for the NGC 1333 brown dwarf candidates assuming a distance of 300 pc. X-ray emitters are denoted by an "X" and non-xray emitters by a solid diamond. The positions of the candidates are shown relative to the theoretical tracks and isochrones of D'Antona \& Mazzitelli $(1994,1997,1998)$ in Fig. 6a, relative to the models of Baraffe et al. (1998) in Fig. 6b, and relative to the models of Burrows et al. $(1997,1998)$ in Fig. 6c. Isochrones from $10^{5}$ years to $10^{8}$ years are shown by solid lines and evolutionary tracks from $0.01 \mathrm{M}_{\odot}$ to $0.40 \mathrm{M}_{\odot}$ are shown by dashed lines. The bold dashed line marks the evolutionary track for a star at the hydrogen-burning limit. The typical error bar for a candidate is shown in the lower left of each plot and is \pm 0.014 dex in $\log \left(\mathrm{T}_{\text {eff }}\right)$ due to uncertainties introduced by the calculation of the $\mathrm{Q}$ index and the fit to derive the dwarf temperature scale. The error of \pm 0.18 dex in $\log \left(\mathrm{L}_{b o l} / \mathrm{L}_{\odot}\right)$ is dominated by uncertainties in the distance $( \pm 30 \mathrm{pc})$ and in the extinction at $\mathrm{J}( \pm 0.37)$. An arrow labeled "250 K" indicates the maximum systematic error introduced by assuming a dwarf temperature scale for a subgiant star.

Fig. 7.- The surface gravities in units of $\mathrm{cm} \mathrm{s}^{-2}$ for the brown dwarf candidates estimated using their masses using the DM tracks. The YSO values are shown by star symbols. For comparison, the surface gravities for dwarf (open triangles) and giant stars (open squares) are from the compilation of Drilling \& Landolt (1999).

Fig. 8.- Spectra for the M giants observed with the IRTF grism. Spectra have been smoothed to a resolution of $\mathrm{R}=170$ for display purposes. The removal of telluric absorption lines, accomplished by dividing a spectrum by that of an AV star, leaves a false emission line at the wavelength of $\mathrm{B} \gamma$ which has been blanked out. The wavelengths of absorption lines of $\mathrm{Na}, \mathrm{Ca}$, and $6 \mathrm{CO}$ bandheads are marked at the bottom of the plot. The three wavelength ranges averaged to compute the water vapor index $\mathrm{Q}$ are labeled at the top of the plot as F1, F2, and F3. 


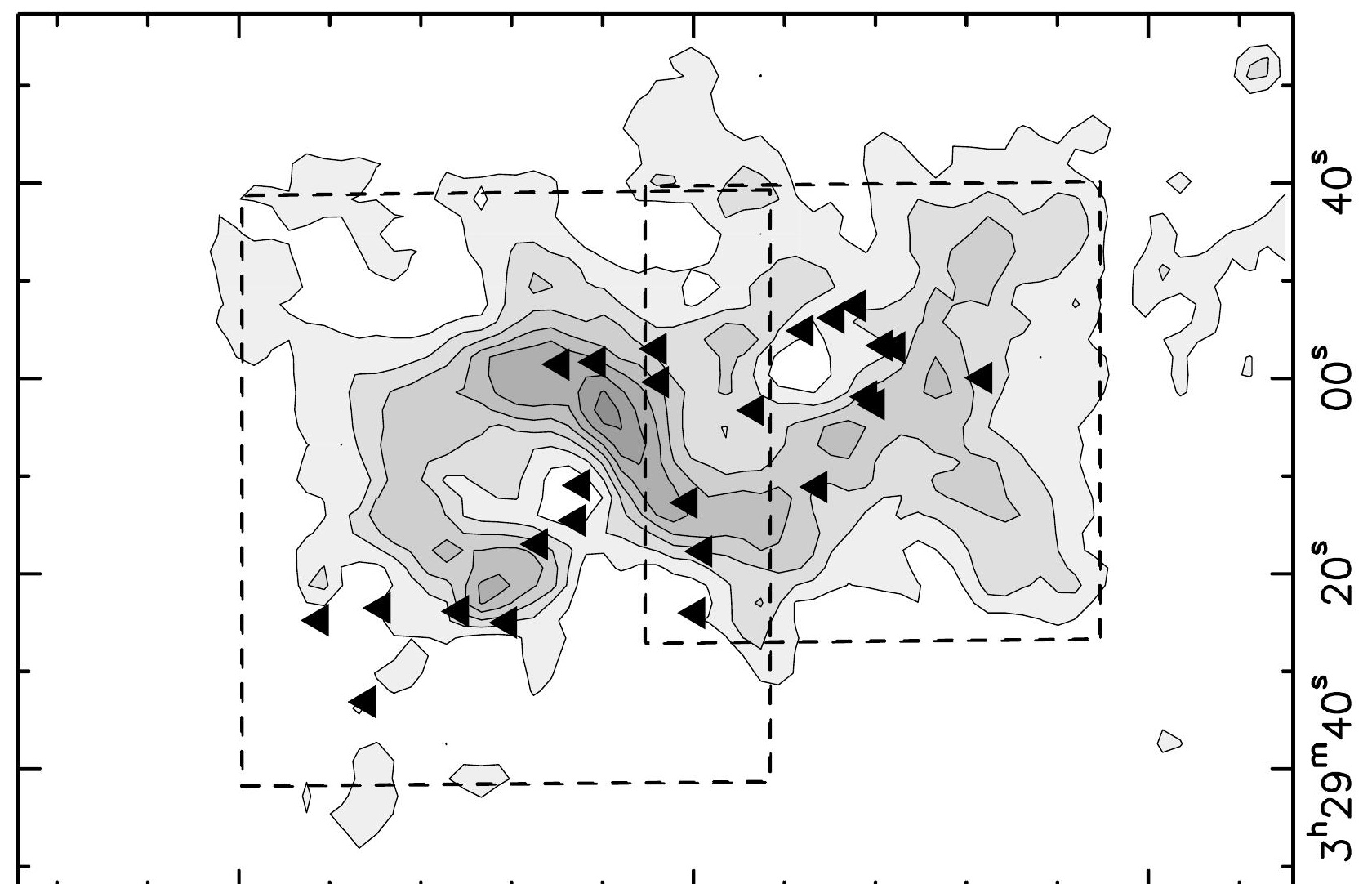




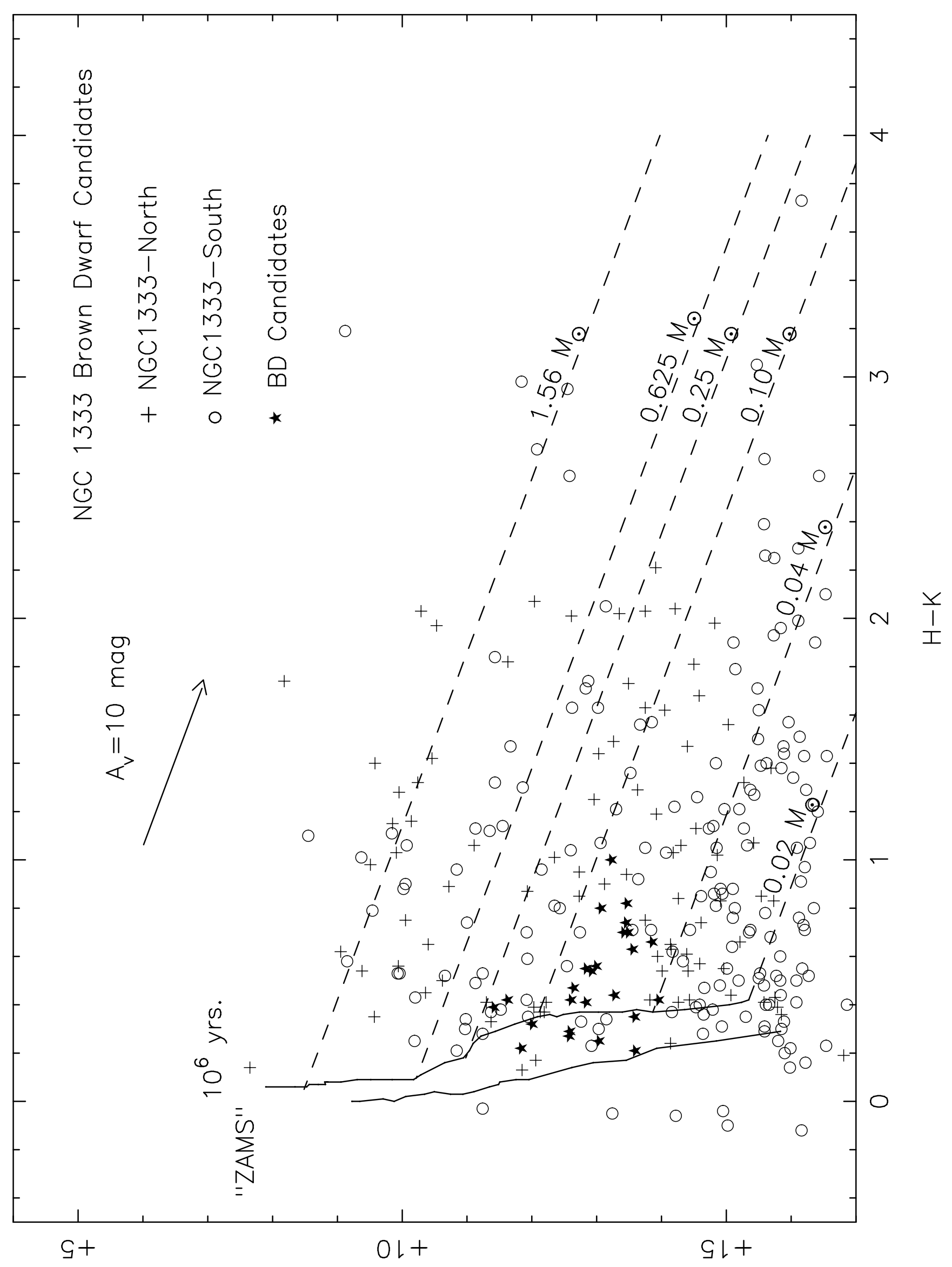




\section{Water Index vs. M Subclass}

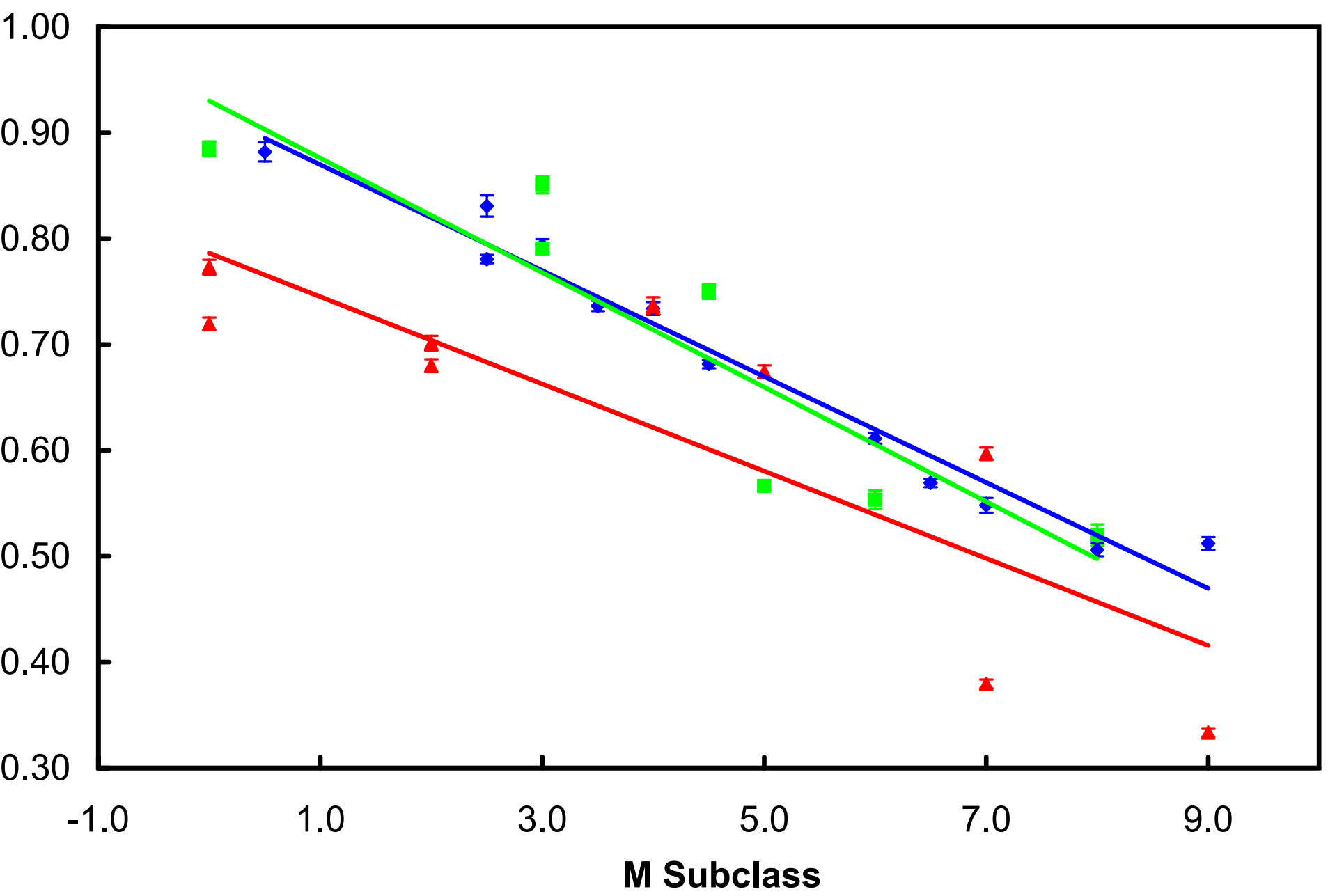




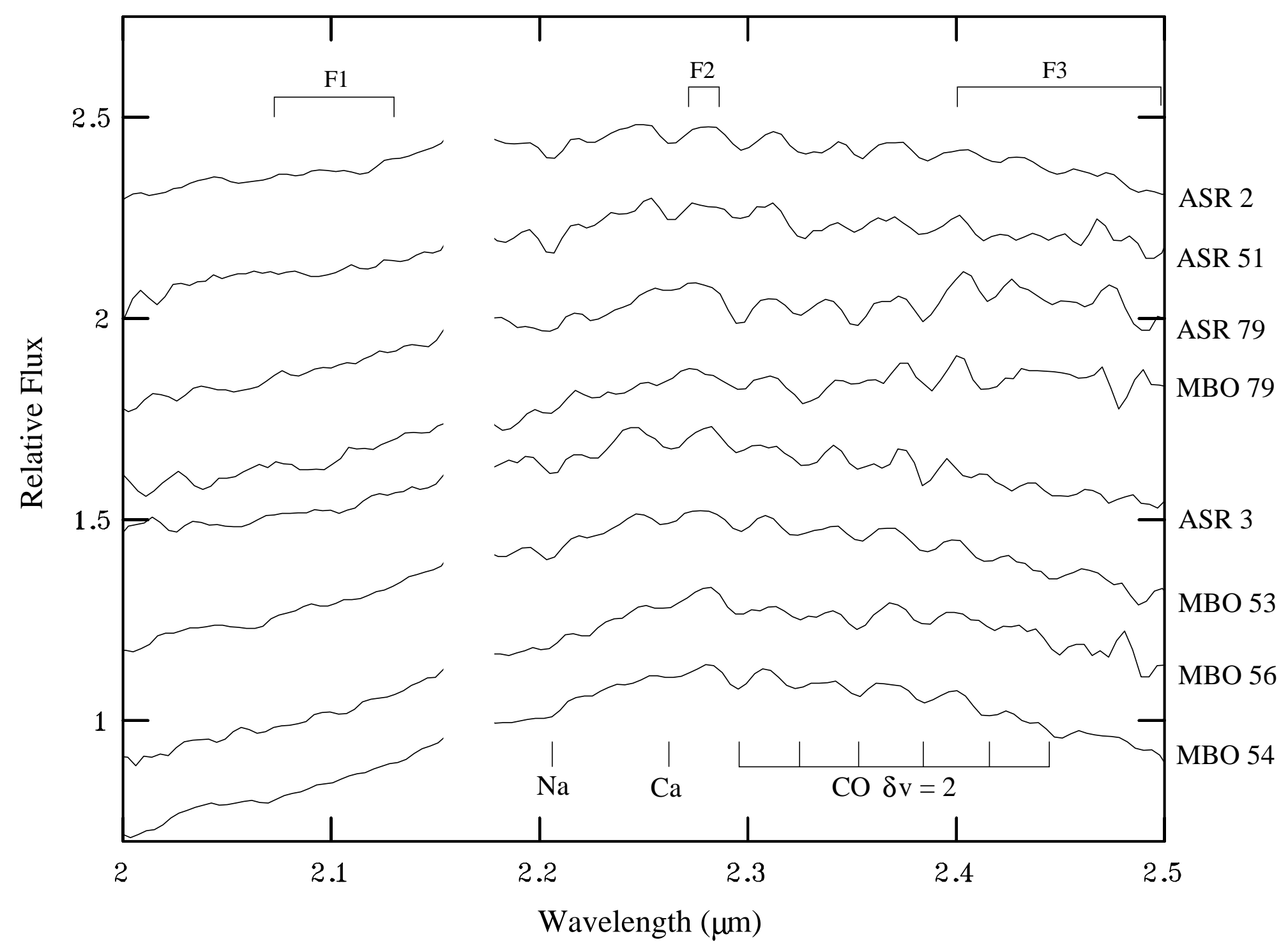




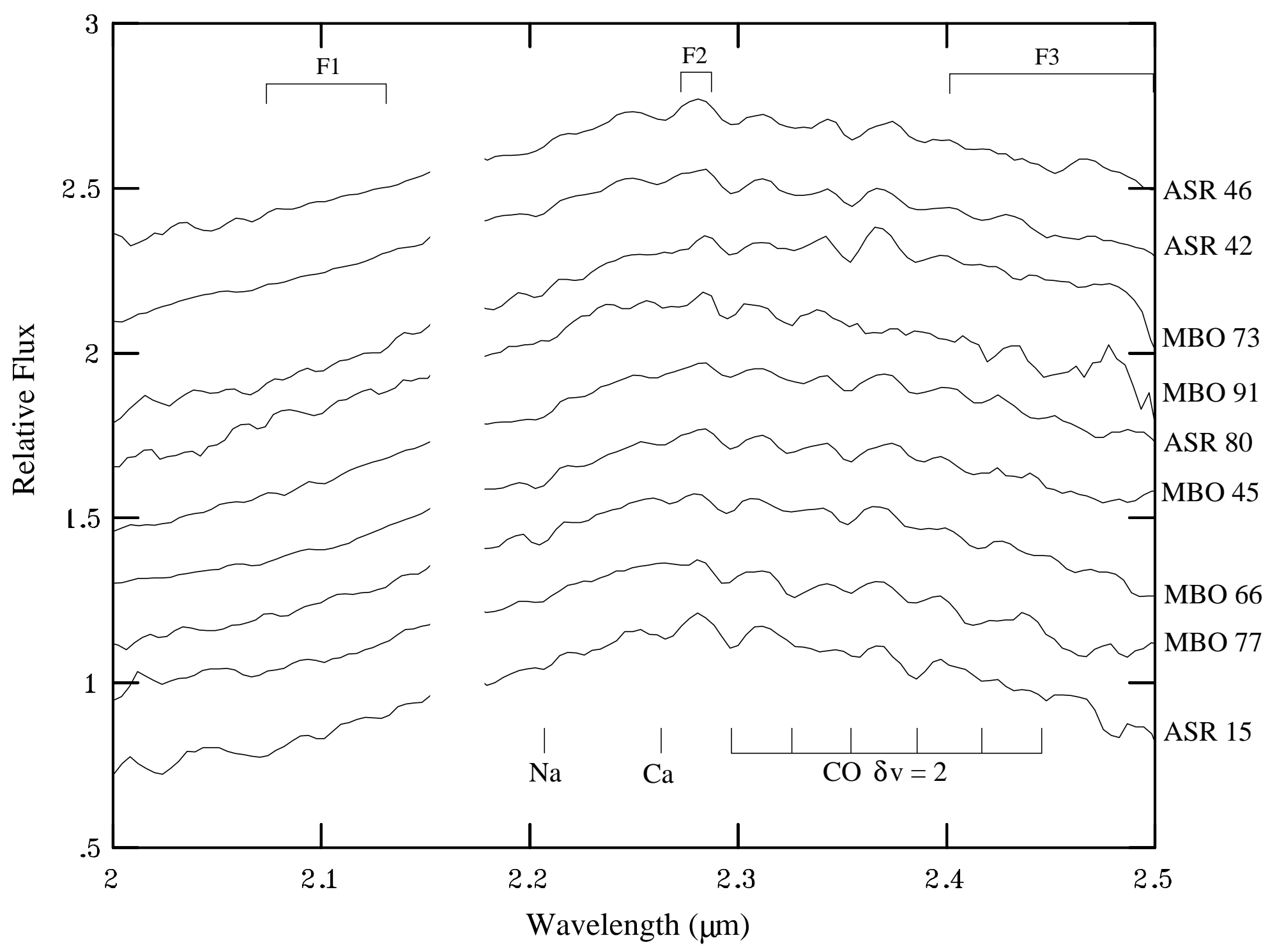




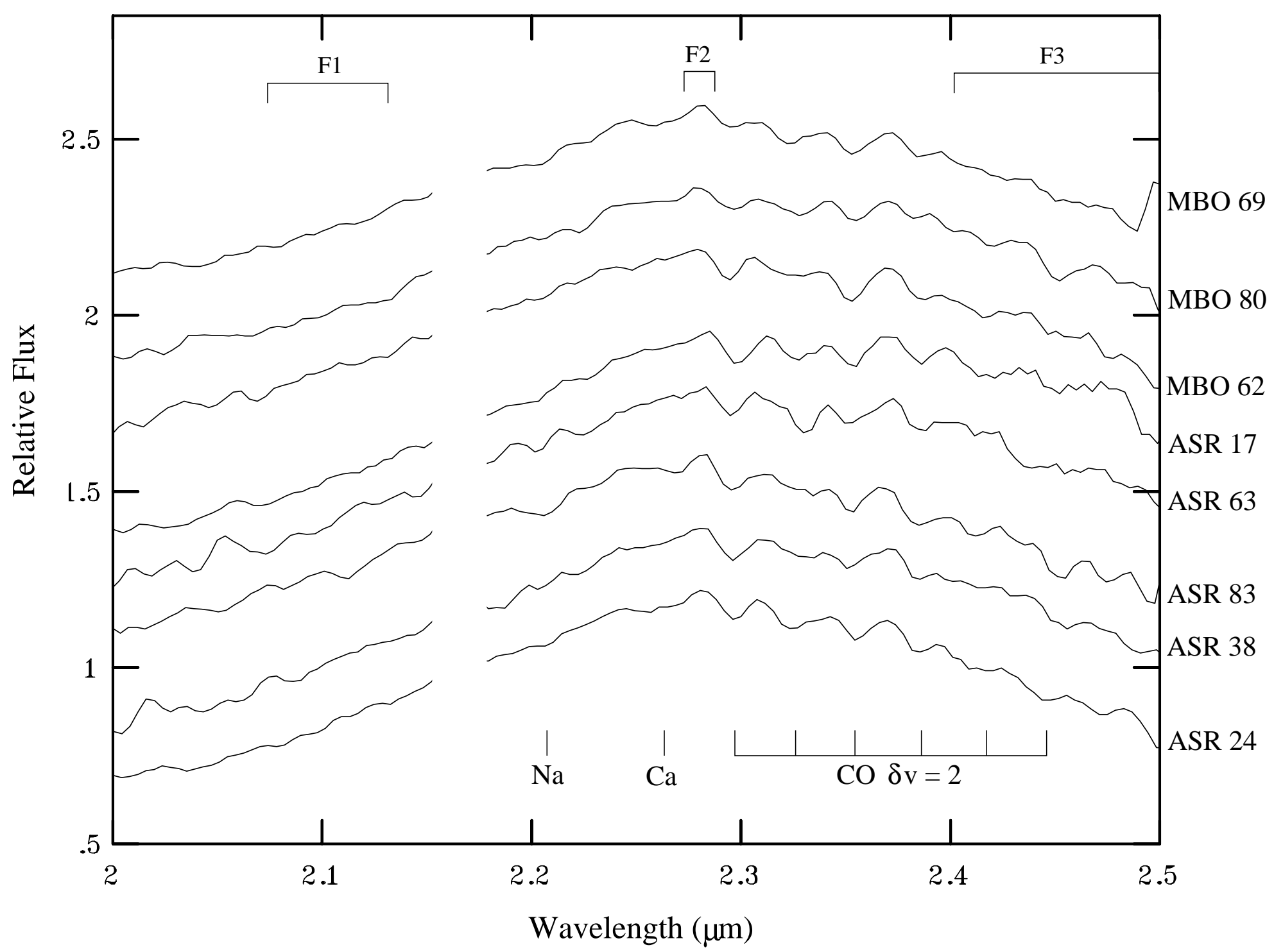




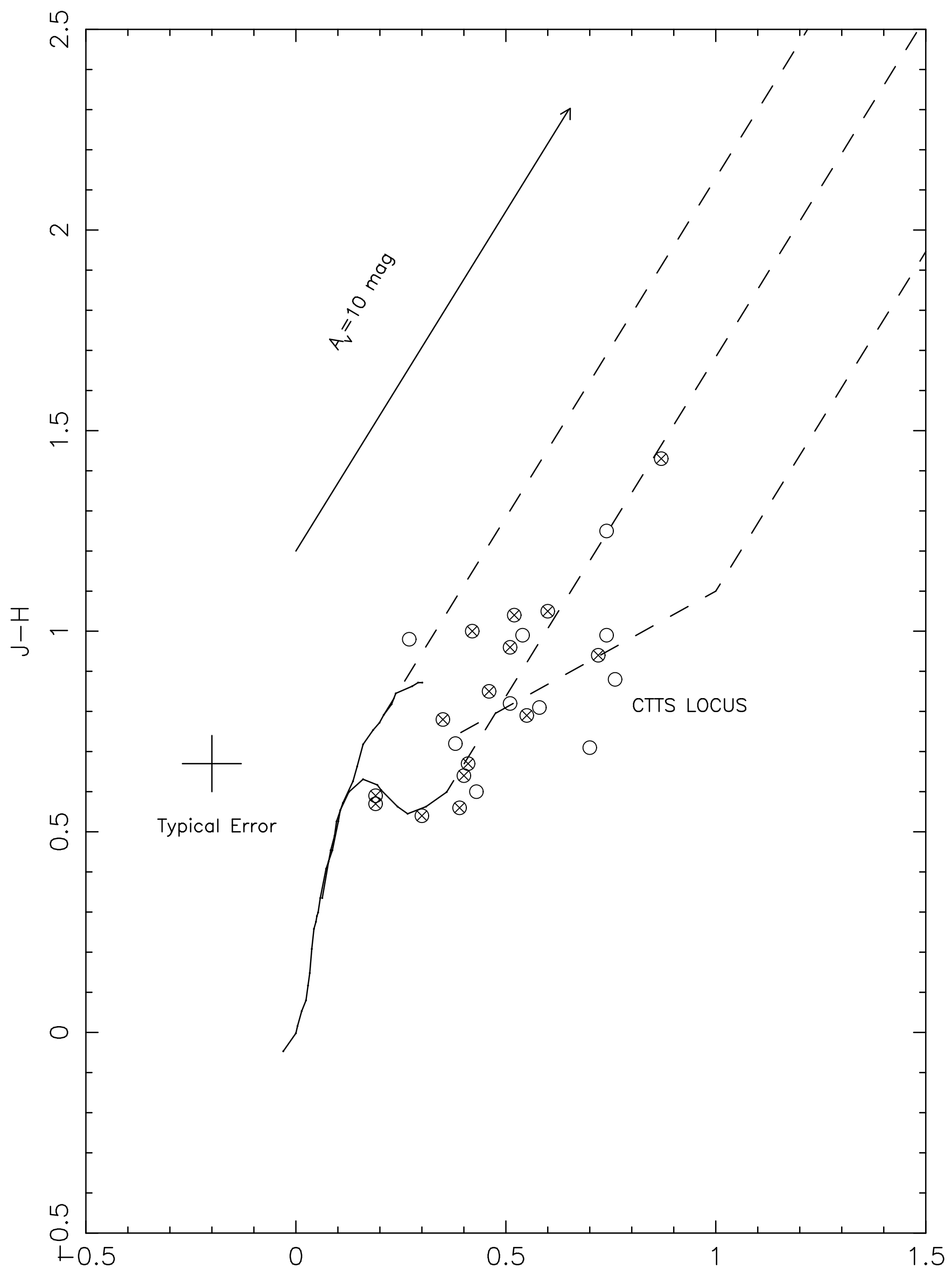




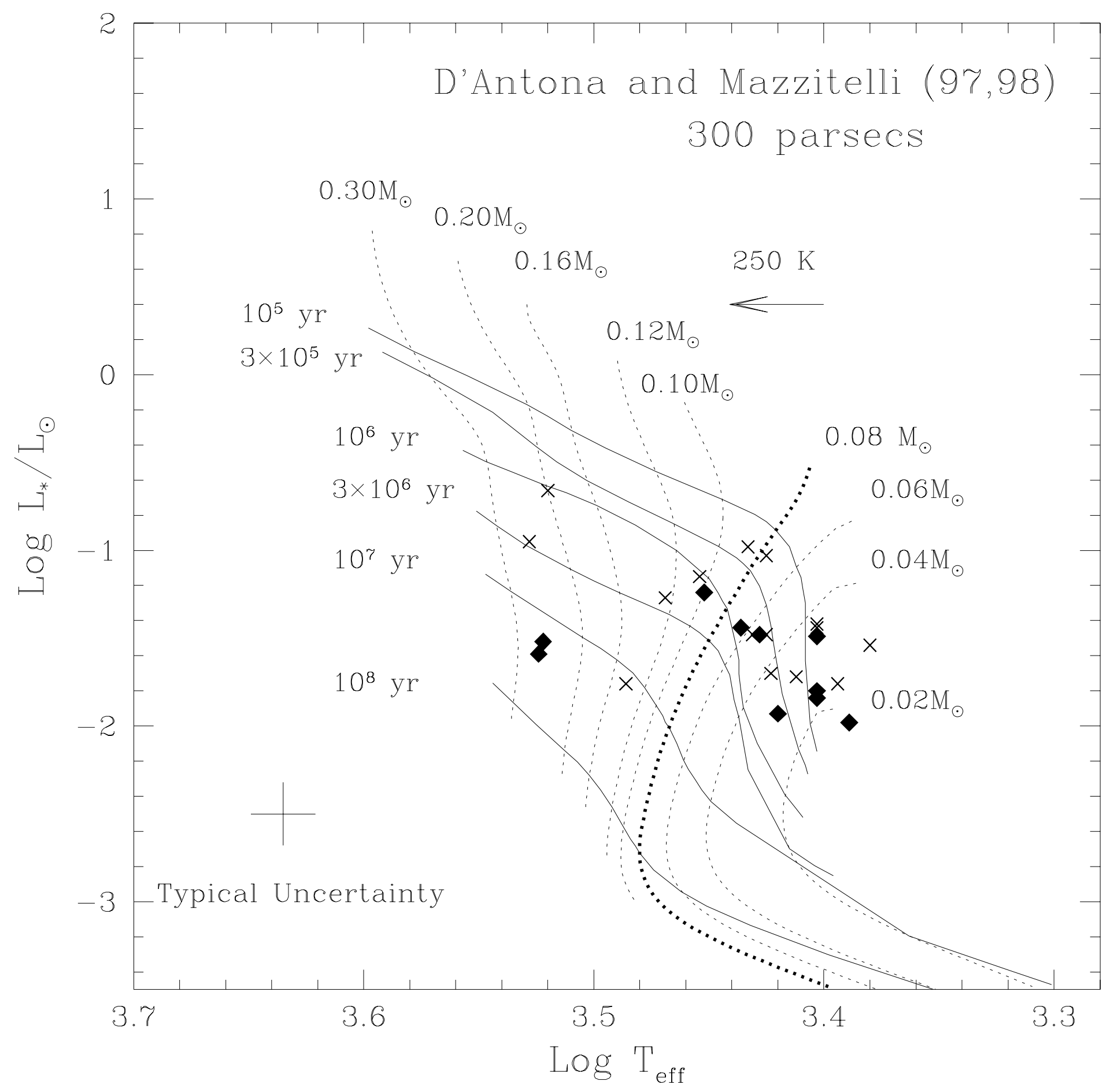




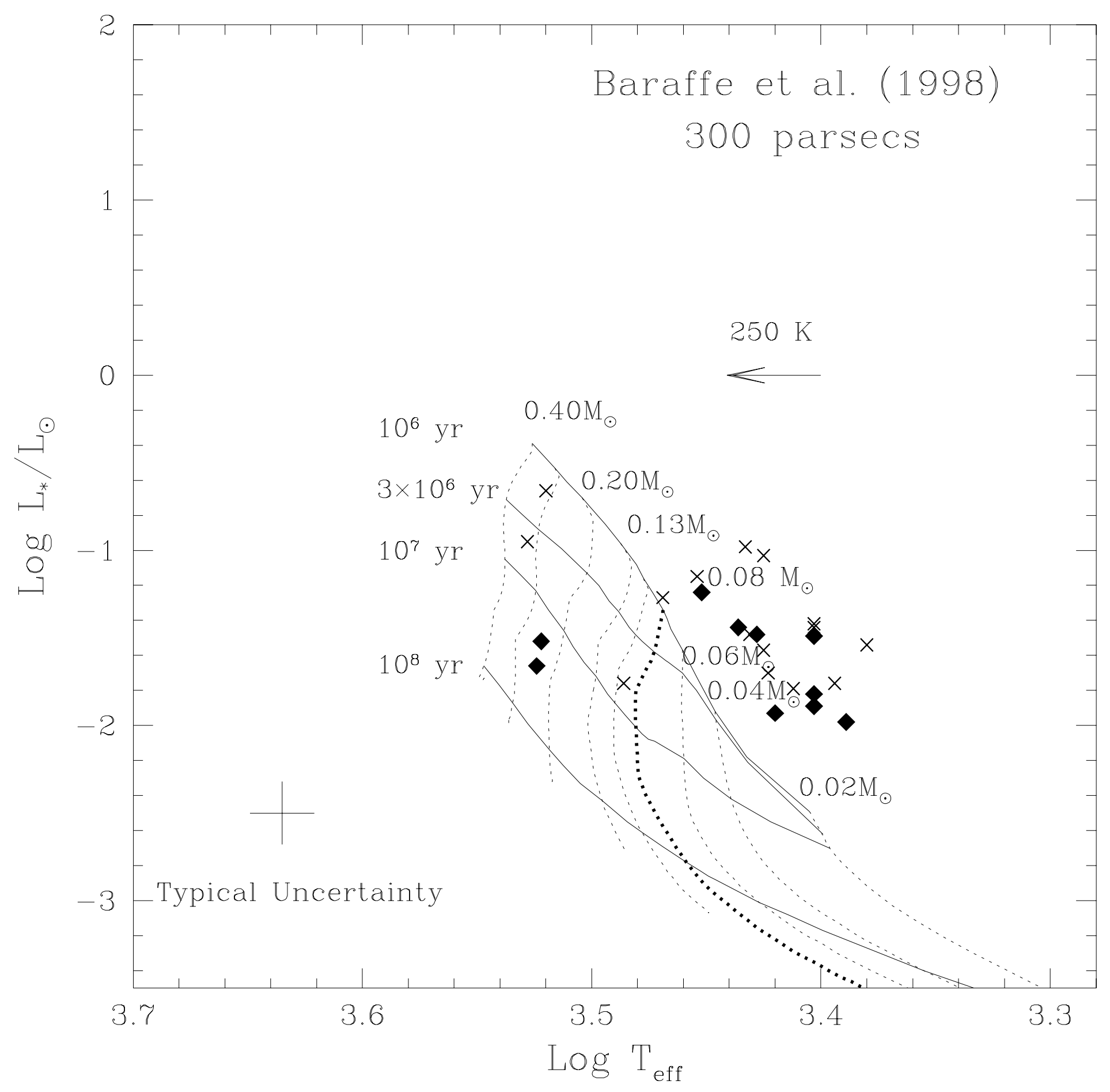




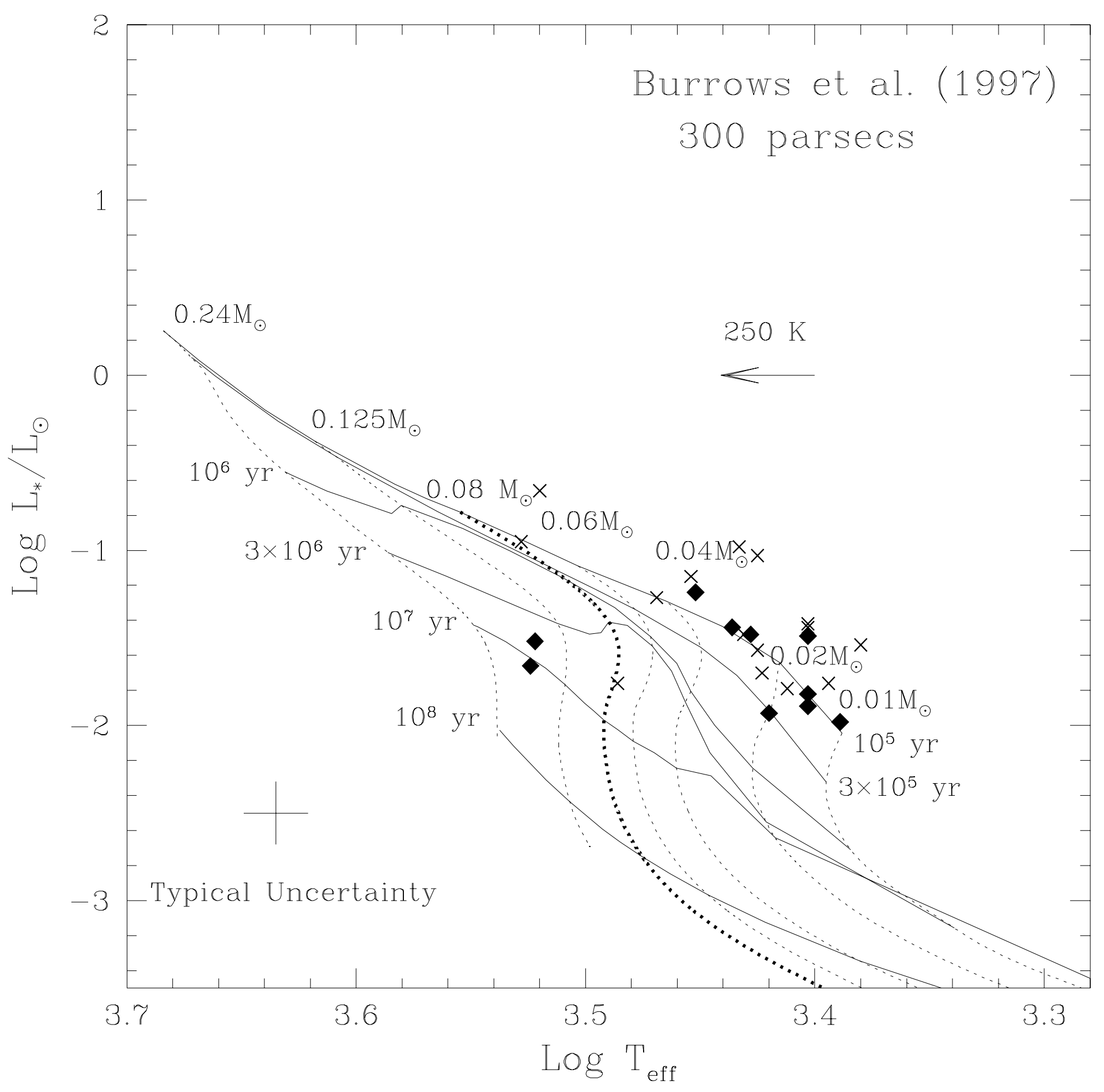


Surface Gravities of Brown Dwarf Candidates

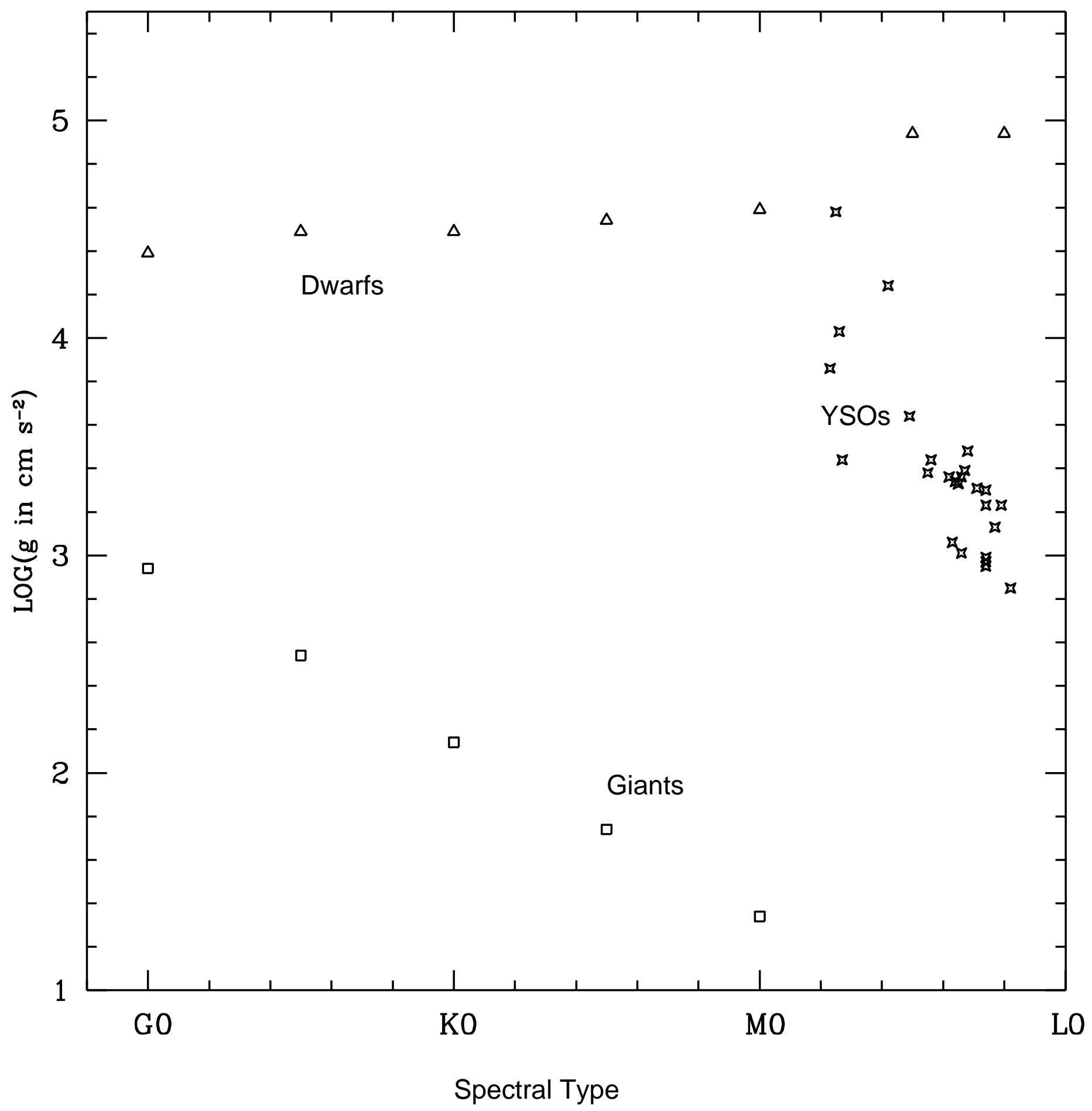




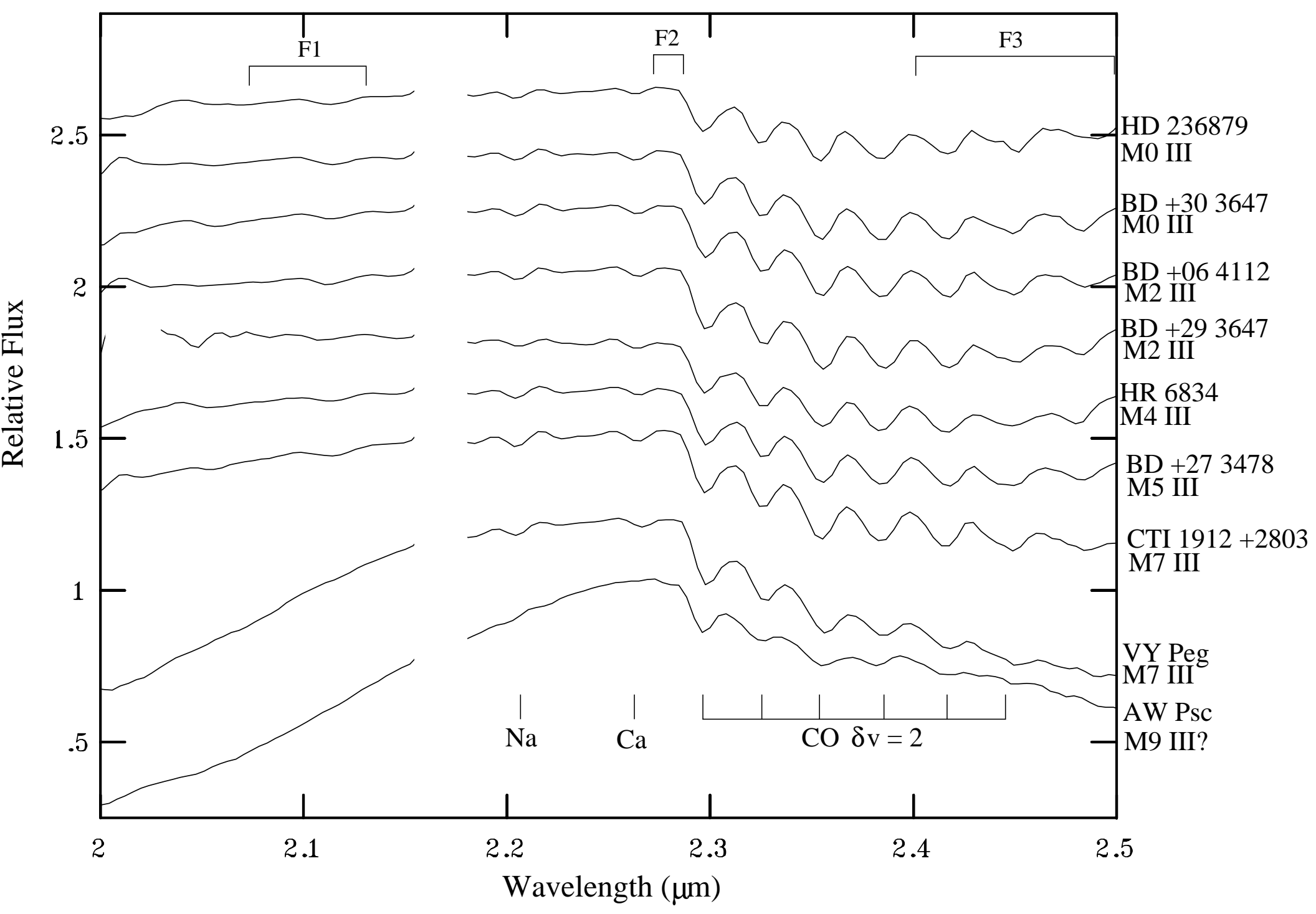


This is Table 1 of our NGC1333 paper 
TABLE 1

Photometry of Northern NGC 1333 Cluster

\begin{tabular}{|c|c|c|c|c|c|c|c|c|c|c|c|}
\hline $\mathrm{MBO}^{\mathrm{a}}$ & $\mathrm{ASR}^{\mathrm{b}}$ & $\mathrm{LAL}^{\mathrm{c}}$ & $\mathrm{RA}(2000)$ & $\mathrm{DEC}(2000)$ & $\mathrm{J}^{\mathrm{d}}$ & $\sigma_{J}^{\mathrm{e}}$ & $\mathrm{H}$ & $\sigma_{H}$ & $\mathrm{~K}$ & $\sigma_{K}$ & Comments \\
\hline 188 & & & 32844.84 & 312929.8 & $\ldots$ & $\ldots$ & 17.65 & 0.21 & 16.04 & 0.10 & \\
\hline 213 & & & $\begin{array}{lll}328 & 46.36\end{array}$ & $\begin{array}{lll}31 & 22 & 32.4\end{array}$ & 18.77 & 0.15 & 17.47 & 0.09 & 16.37 & 0.12 & \\
\hline 217 & & & 32846.69 & $\begin{array}{lll}31 & 26 & 31.7\end{array}$ & 18.80 & 0.14 & 17.72 & 0.12 & 16.49 & 0.12 & \\
\hline 142 & 89 & 91 & $328 \quad 46.64$ & $\begin{array}{lll}31 & 19 & 49.8\end{array}$ & 17.57 & 0.06 & 16.20 & 0.04 & 15.41 & 0.06 & \\
\hline 92 & 88 & 92 & 32846.85 & $\begin{array}{lll}31 & 20 & 27.4\end{array}$ & 14.71 & 0.01 & 14.51 & 0.01 & 13.97 & 0.02 & \\
\hline 206 & & & $\begin{array}{lll}3 & 28 & 47.17\end{array}$ & 312434.6 & 18.14 & 0.08 & 17.02 & 0.06 & 16.27 & 0.09 & \\
\hline 221 & 66 & & $\begin{array}{lll}328 & 47.15\end{array}$ & $\begin{array}{lll}31 & 18 & 45.5\end{array}$ & $\ldots$ & $\ldots$ & 17.32 & 0.11 & 15.56 & 0.08 & \\
\hline 221 & & & $\begin{array}{lll}328 & 47.25\end{array}$ & $\begin{array}{lll}31 & 23 & 10.0\end{array}$ & $\ldots$ & $\ldots$ & 18.09 & 0.24 & 16.63 & 0.14 & \\
\hline 34 & & 93 & $\begin{array}{lll}328 & 47.62\end{array}$ & 312406.0 & 14.47 & 0.00 & 12.87 & 0.00 & 11.51 & 0.00 & \\
\hline 139 & & & $328 \quad 47.64$ & 312154.4 & 17.73 & 0.06 & 16.51 & 0.04 & 15.35 & 0.05 & \\
\hline 180 & & & 32848.02 & 312429.6 & 18.65 & 0.13 & 17.35 & 0.08 & 15.93 & 0.07 & \\
\hline 117 & 65 & 99 & $\begin{array}{lll}328 & 48.14\end{array}$ & $\begin{array}{lll}31 & 19 & 23.1\end{array}$ & 15.41 & 0.01 & 15.19 & 0.02 & 14.88 & 0.04 & \\
\hline 100 & 90 & 100 & $\begin{array}{lll}3 & 28 & 48.44\end{array}$ & 312028.2 & 17.12 & 0.03 & 15.50 & 0.02 & 14.33 & 0.02 & \\
\hline 82 & & & $328 \quad 49.45$ & 312506.1 & 15.73 & 0.01 & 14.35 & 0.01 & 13.63 & 0.01 & \\
\hline 95 & & & $328 \quad 49.69$ & 312453.0 & 16.02 & 0.01 & 14.88 & 0.01 & 14.25 & 0.02 & \\
\hline 26 & & & $\begin{array}{lll}3 & 28 & 50.93\end{array}$ & $\begin{array}{lll}31 & 23 & 47.9\end{array}$ & 14.55 & 0.01 & 12.37 & 0.00 & 11.21 & 0.00 & \\
\hline 6 & 122 & 106 & $\begin{array}{lll}328 & 50.98\end{array}$ & $\begin{array}{lll}31 & 18 & 17.9\end{array}$ & 10.73 & 0.00 & 10.38 & 0.00 & 9.48: & 0.00 & SVS 10 , edge of frame at $K$ \\
\hline 14 & 125 & 110 & $328 \quad 51.15$ & $31 \quad 1954.5$ & 11.71 & 0.00 & 10.87 & 0.00 & 10.21 & 0.00 & \\
\hline 122 & & 114 & $\begin{array}{lll}328 & 51.73\end{array}$ & $\begin{array}{lll}31 & 26 & 09.6\end{array}$ & 16.34 & 0.02 & 15.47 & 0.02 & 14.98 & 0.03 & \\
\hline 18 & & 120 & $\begin{array}{lll}3 & 28 & 52.13\end{array}$ & $\begin{array}{lll}31 & 22 & 45.3\end{array}$ & 12.05 & 0.00 & 11.27 & 0.00 & 10.74 & 0.00 & \\
\hline 144 & & & $\begin{array}{lll}3 & 28 & 52.61\end{array}$ & $\begin{array}{lll}31 & 22 & 01.2\end{array}$ & 19.35 & 0.24 & 16.77 & 0.06 & 15.45 & 0.05 & \\
\hline 145 & & & $\begin{array}{lll}3 & 28 & 54.57\end{array}$ & $\begin{array}{lll}31 & 27 & 33.1\end{array}$ & 17.09 & 0.03 & 16.18 & 0.02 & 15.45 & 0.03 & \\
\hline 50 & & 139 & 32855.19 & 312522.0 & 14.52 & 0.00 & 13.19 & 0.00 & 12.62 & 0.01 & \\
\hline 194 & & & $328 \quad 55.31$ & $\begin{array}{lll}31 & 26 & 13.9\end{array}$ & 17.25 & 0.03 & 16.67 & 0.03 & 16.16 & 0.06 & \\
\hline 112 & & & $328 \quad 55.48$ & $\begin{array}{lll}31 & 26 & 43.7\end{array}$ & 16.03 & 0.01 & 15.22 & 0.01 & 14.74 & 0.02 & \\
\hline 106 & & 144 & $\begin{array}{lll}328 & 56.02\end{array}$ & $\begin{array}{lll}31 & 26 & 33.5\end{array}$ & 15.95 & 0.01 & 15.08 & 0.01 & 14.67 & 0.02 & \\
\hline 220 & & & $328 \quad 56.07$ & $\begin{array}{lll}31 & 26 & 50.5\end{array}$ & 17.74 & 0.04 & 16.86 & 0.04 & 16.62 & 0.09 & \\
\hline 146 & & & $\begin{array}{lll}328 & 56.07\end{array}$ & $\begin{array}{lll}31 & 19 & 08.6\end{array}$ & 19.44 & 0.19 & 18.23 & 0.14 & 16.19 & 0.07 & \\
\hline 125 & & & 32856.30 & 312442.8 & 17.60 & 0.04 & 15.90 & 0.02 & 15.02 & 0.03 & \\
\hline 37 & & 147 & $328 \quad 56.27$ & $\begin{array}{lll}31 & 22 & 27.9\end{array}$ & 15.55 & 0.01 & 13.26 & 0.00 & 11.75 & 0.00 & \\
\hline 11 & 120 & 150 & $\begin{array}{lll}328 & 56.58\end{array}$ & $\begin{array}{llll}31 & 18 & 35.5\end{array}$ & 12.15 & 0.00 & 10.99 & 0.00 & 10.10 & 0.00 & SVS 11 \\
\hline 107 & 124 & 151 & $\begin{array}{lll}3 & 28 & 56.82\end{array}$ & $\begin{array}{lll}31 & 19 & 45.6\end{array}$ & 16.04 & 0.02 & 15.21 & 0.02 & 14.67 & 0.03 & \\
\hline 91 & & 152 & $\begin{array}{lll}3 & 28 & 56.91\end{array}$ & 312048.6 & 15.33 & 0.01 & 14.62 & 0.01 & 13.94 & 0.01 & \\
\hline 199 & & & $\begin{array}{lll}328 & 57.09\end{array}$ & 312124.7 & $\ldots$ & $\ldots$ & 18.54 & 0.23 & 16.19 & 0.09 & \\
\hline 148 & 64 & & $\begin{array}{lll}328 & 57.08\end{array}$ & $\begin{array}{lll}31 & 19 & 12.0\end{array}$ & 17.13 & 0.03 & 16.10 & 0.03 & 15.54 & 0.06 & \\
\hline 21 & 115 & 158 & $328 \quad 57.36$ & $\begin{array}{lll}31 & 19 & 50.5\end{array}$ & 11.90 & 0.00 & 11.13 & 0.00 & 10.92 & 0.00 & $4.7^{\prime \prime}$ from $\mathrm{MBO} 35$ \\
\hline 134 & & & $\begin{array}{lll}328 & 57.50\end{array}$ & 312012.1 & $\ldots$ & $\ldots$ & 17.15 & 0.10 & 15.19 & 0.06 & neb. in $\mathrm{HH} 12$ complex \\
\hline 35 & 113 & & $328 \quad 57.64$ & $\begin{array}{lll}31 & 19 & 48.2\end{array}$ & 12.82 & 0.00 & 11.99 & 0.00 & 11.60 & 0.00 & $4.7^{\prime \prime}$ from MBO 21 \\
\hline 73 & & 164 & $\begin{array}{lll}3 & 28 & 58.22\end{array}$ & $\begin{array}{lll}31 & 22 & 09.4\end{array}$ & 15.98 & 0.01 & 14.34 & 0.01 & 13.31 & 0.01 & \\
\hline 47 & & 163 & $\begin{array}{lll}328 & 58.23\end{array}$ & $\begin{array}{lll}31 & 22 & 02.2\end{array}$ & 15.02 & 0.01 & 13.22 & 0.00 & 12.23 & 0.01 & \\
\hline 170 & & & $\begin{array}{lll}328 & 58.30\end{array}$ & $\begin{array}{llll}31 & 20 & 27.7\end{array}$ & $\ldots$ & $\ldots$ & $\ldots$ & $\ldots$ & 15.76 & 0.15 & neb. in HH 12 complex \\
\hline 80 & & 165 & $\begin{array}{lll}3 & 28 & 58.39\end{array}$ & 312256.8 & 15.23 & 0.01 & 14.29 & 0.01 & 13.57 & 0.01 & \\
\hline 38 & & 166 & $\begin{array}{lll}3 & 28 & 58.38\end{array}$ & $\begin{array}{lll}31 & 22 & 17.6\end{array}$ & 18.60 & 0.09 & 14.98 & 0.01 & 11.92 & 0.00 & \\
\hline 192 & & & $328 \quad 58.90$ & $\begin{array}{lll}31 & 29 & 06.7\end{array}$ & $\ldots$ & $\ldots$ & 17.48 & 0.10 & 16.11 & 0.07 & \\
\hline 113 & & & $\begin{array}{lll}3 & 28 & 59.18\end{array}$ & $\begin{array}{llll}31 & 20 & 32.3\end{array}$ & 16.52 & 0.02 & 15.97 & 0.03 & 14.81 & 0.08 & star in $\mathrm{HH} 12$ neb. \\
\hline 123 & & & $328 \quad 59.45$ & $\begin{array}{lll}31 & 29 & 30.6\end{array}$ & 17.37 & 0.05 & 15.89 & 0.03 & 14.99 & 0.03 & \\
\hline 102 & 116 & 170 & $\begin{array}{lll}3 & 28 & 59.30\end{array}$ & $\begin{array}{lll}31 & 20 & 08.3\end{array}$ & 17.61 & 0.06 & 17.21 & 0.11 & 14.43 & 0.04 & \\
\hline 12 & & 173 & $\begin{array}{lll}3 & 28 & 59.52\end{array}$ & $\begin{array}{lll}31 & 21 & 46.9\end{array}$ & 13.05 & 0.00 & 11.05 & 0.00 & 10.13 & 0.00 & \\
\hline 78 & & 175 & $\begin{array}{lll}3 & 29 & 00.12\end{array}$ & $\begin{array}{lll}31 & 21 & 09.3\end{array}$ & 16.44 & 0.02 & 14.63 & 0.01 & 13.38 & 0.01 & \\
\hline 83 & & 176 & $\begin{array}{lll}3 & 29 & 00.34\end{array}$ & $\begin{array}{lll}31 & 24 & 38.4\end{array}$ & 16.55 & 0.02 & 14.66 & 0.01 & 13.72 & 0.01 & \\
\hline 45 & 133 & 177 & 32900.34 & $\begin{array}{lll}31 & 20 & 45.7\end{array}$ & 13.35 & 0.00 & 12.41 & 0.00 & 12.09 & 0.01 & \\
\hline 149 & & & $\begin{array}{lll}3 & 29 & 00.44\end{array}$ & 312404.1 & $\ldots$ & $\ldots$ & 18.68 & 0.24 & 15.55 & 0.04 & nebulous \\
\hline 39 & & 180 & $\begin{array}{lll}329 & 00.68\end{array}$ & $\begin{array}{lll}31 & 22 & 01.0\end{array}$ & 16.12 & 0.01 & 13.27 & 0.00 & 11.94 & 0.00 & \\
\hline 209 & & & $\begin{array}{lll}3 & 29 & 01.33\end{array}$ & $\begin{array}{lll}31 & 19 & 35.6\end{array}$ & 18.80 & 0.16 & 17.03 & 0.09 & 16.29 & 0.12 & \\
\hline 219 & & & 32901.44 & $3120 \quad 55.3$ & $\ldots$ & $\ldots$ & 18.76 & 0.28 & 16.61 & 0.11 & \\
\hline 150 & & & 32901.55 & $\begin{array}{lll}31 & 25 & 13.1\end{array}$ & 18.92 & 0.13 & 17.10 & 0.05 & 15.57 & 0.04 & \\
\hline 19 & 114 & 181 & 32901.50 & $\begin{array}{lll}31 & 20 & 20.8\end{array}$ & 17.49 & 0.04 & 14.01 & 0.01 & 10.84 & 0.00 & SVS 12 ,ext. neb. to NW \\
\hline 138 & & & $\begin{array}{lll}3 & 29 & 02.02\end{array}$ & $\begin{array}{lll}31 & 25 & 09.4\end{array}$ & 18.40 & 0.09 & 16.52 & 0.04 & 15.28 & 0.04 & \\
\hline 169 & & & $\begin{array}{lll}32 & 29 & 02.06\end{array}$ & 312631.0 & 18.59 & 0.09 & 16.45 & 0.03 & 15.76 & 0.05 & \\
\hline 208 & & & $\begin{array}{lll}3 & 29 & 02.55\end{array}$ & $\begin{array}{lll}31 & 26 & 02.3\end{array}$ & $\ldots$ & $\ldots$ & 17.28 & 0.06 & 16.29 & 0.08 & \\
\hline 84 & 62 & 187 & $\begin{array}{lll}3 & 29 & 02.64\end{array}$ & $\begin{array}{lll}31 & 19 & 06.1\end{array}$ & 17.84 & 0.06 & 15.39 & 0.02 & 13.73 & 0.01 & \\
\hline 67 & & & $\begin{array}{lll}3 & 29 & 02.75\end{array}$ & $\begin{array}{lll}31 & 22 & 17.4\end{array}$ & 16.82 & 0.03 & 14.77 & 0.01 & 13.10 & 0.01 & \\
\hline 31 & & 189 & $\begin{array}{lll}3 & 29 & 03.08\end{array}$ & $\begin{array}{lll}31 & 22 & 38.3\end{array}$ & 14.15 & 0.00 & 12.58 & 0.00 & 11.43 & 0.00 & \\
\hline 89 & & 190 & $\begin{array}{lll}329 & 03.16\end{array}$ & 312544.9 & 15.57 & 0.01 & 14.65 & 0.01 & 13.92 & 0.01 & \\
\hline
\end{tabular}


TABLE 1-Continued

\begin{tabular}{|c|c|c|c|c|c|c|c|c|c|c|c|}
\hline $\mathrm{MBO}^{\mathrm{a}}$ & $\mathrm{ASR}^{\mathrm{b}}$ & $\mathrm{LAL}^{\mathrm{c}}$ & $\mathrm{RA}(2000)$ & $\operatorname{DEC}(2000)$ & $\mathrm{J}^{\mathrm{d}}$ & $\sigma_{J}^{\mathrm{e}}$ & $\mathrm{H}$ & $\sigma_{H}$ & $\mathrm{~K}$ & $\sigma_{K}$ & Comments \\
\hline 90 & & 191 & $\begin{array}{lll}32 & 29 & 03.27\end{array}$ & $\begin{array}{lll}31 & 23 & 14.8\end{array}$ & 17.49 & 0.04 & 15.54 & 0.02 & 13.93 & 0.01 & \\
\hline 87 & & 192 & 32903.38 & 312514.1 & 16.29 & 0.02 & 14.91 & 0.01 & 13.83 & 0.01 & \\
\hline 88 & 63 & 193 & 32903.34 & $31 \quad 18 \quad 40.0$ & 15.60 & 0.01 & 14.61 & 0.01 & 13.87 & 0.02 & \\
\hline 105 & & & $\begin{array}{lll}3 & 29 & 03.74\end{array}$ & $\begin{array}{lll}31 & 23 & 08.8\end{array}$ & 17.80 & 0.06 & 15.93 & 0.02 & 14.63 & 0.02 & \\
\hline 5 & & 195 & $\begin{array}{lll}3 & 29 & 03.80\end{array}$ & $\begin{array}{lll}31 & 21 & 48.9\end{array}$ & 11.40 & 0.00 & 10.48 & 0.00 & 9.45 & 0.00 & SVS 8 \\
\hline 116 & & & $\begin{array}{lll}3 & 29 & 03.89\end{array}$ & $\begin{array}{lll}31 & 23 & 31.0\end{array}$ & 17.17 & 0.04 & 16.05 & 0.02 & 14.88 & 0.03 & \\
\hline 20 & & 198 & $\begin{array}{lll}3 & 29 & 04.12\end{array}$ & $\begin{array}{lll}31 & 25 & 14.8\end{array}$ & 13.48 & 0.00 & 11.90 & 0.00 & 10.92 & 0.00 & \\
\hline 151 & & & $\begin{array}{lll}3 & 29 & 04.41\end{array}$ & $\begin{array}{lll}31 & 28 & 03.4\end{array}$ & 17.47 & 0.04 & 16.10 & 0.02 & 15.58 & 0.04 & \\
\hline 156 & 61 & & $\begin{array}{lll}3 & 29 & 04.25\end{array}$ & $\begin{array}{lll}31 & 19 & 06.7\end{array}$ & 16.39 & 0.02 & 16.38 & 0.04 & 15.64 & 0.06 & \\
\hline 86 & & 202 & $\begin{array}{lll}32 & 29 & 04.57\end{array}$ & $\begin{array}{lll}31 & 20 & 29.1\end{array}$ & 17.42 & 0.05 & 15.34 & 0.02 & 13.75 & 0.01 & \\
\hline 63 & 87 & 204 & $\begin{array}{lll}32 & 29 & 04.88\end{array}$ & $\begin{array}{lll}31 & 20 & 38.7\end{array}$ & 16.73 & 0.05 & 14.74 & 0.02 & 12.95 & 0.01 & ext. neb. to SE \\
\hline 152 & & & 32905.21 & 312647.0 & 19.71 & 0.25 & 17.25 & 0.05 & 15.58 & 0.04 & \\
\hline 71 & & 206 & $\begin{array}{lll}32 & 29 & 05.62\end{array}$ & $\begin{array}{lll}31 & 21 & 34.1\end{array}$ & 18.01 & 0.09 & 15.32 & 0.01 & 13.22 & 0.01 & \\
\hline 143 & & & $\begin{array}{lll}32 & 295.59\end{array}$ & $\begin{array}{lll}31 & 20 & 10.9\end{array}$ & 17.10 & 0.03 & 16.15 & 0.03 & 15.43 & 0.05 & \\
\hline 215 & & & $\begin{array}{lll}32 & 29 & 05.73\end{array}$ & $\begin{array}{lll}31 & 25 & 21.7\end{array}$ & 18.76 & 0.13 & 17.69 & 0.10 & 16.43 & 0.09 & \\
\hline 93 & & 210 & $\begin{array}{lll}3 & 29 & 06.74\end{array}$ & $\begin{array}{lll}31 & 22 & 58.5\end{array}$ & 16.82 & 0.04 & 15.21 & 0.01 & 14.15 & 0.02 & \\
\hline 141 & & & $\begin{array}{lll}3 & 29 & 07.11\end{array}$ & $\begin{array}{lll}31 & 23 & 22.8\end{array}$ & 17.85 & 0.07 & 16.48 & 0.04 & 15.40 & 0.04 & \\
\hline 216 & & & $\begin{array}{lll}3 & 29 & 07.19\end{array}$ & $\begin{array}{lll}31 & 24 & 09.9\end{array}$ & $\ldots$ & $\ldots$ & 18.39 & 0.22 & 16.45 & 0.09 & \\
\hline 132 & & & $\begin{array}{lll}3 & 29 & 07.59\end{array}$ & 312426.6 & 17.54 & 0.04 & 15.95 & 0.02 & 15.18 & 0.03 & \\
\hline 3 & & 213 & 32907.70 & 312157.5 & 16.47 & 0.11 & 12.47 & 0.01 & $9.20:$ & 0.00 & sat. at $\mathrm{K}$ ?,neb. \\
\hline 13 & & 215 & $\begin{array}{lll}3 & 29 & 07.89\end{array}$ & 312251.6 & 12.92 & 0.00 & 11.24 & 0.00 & 10.15 & 0.00 & \\
\hline 205 & & & 32908.00 & $\begin{array}{lll}31 & 27 & 27.4\end{array}$ & 17.65 & 0.04 & 16.81 & 0.04 & 16.25 & 0.06 & \\
\hline 44 & 86 & 216 & $\begin{array}{lll}3 & 29 & 08.28\end{array}$ & 312020.6 & 14.45 & 0.00 & 12.98 & 0.00 & 12.07 & 0.00 & \\
\hline 96 & & 219 & $\begin{array}{lll}32 & 298.58\end{array}$ & $\begin{array}{lll}31 & 22 & 29.6\end{array}$ & 17.46 & 0.07 & 15.53 & 0.03 & 14.28 & 0.03 & \\
\hline 108 & & 220 & 32908.93 & $\begin{array}{lll}31 & 26 & 23.4\end{array}$ & 16.64 & 0.02 & 15.57 & 0.01 & 14.69 & 0.02 & \\
\hline 33 & & 221 & 32908.91 & $\begin{array}{lll}31 & 22 & 56.3\end{array}$ & 16.26 & 0.02 & 13.39 & 0.00 & 11.51 & 0.00 & \\
\hline 36 & & 222 & $\begin{array}{lll}3 & 29 & 09.03\end{array}$ & $\begin{array}{lll}31 & 23 & 05.8\end{array}$ & 14.46 & 0.01 & 12.80 & 0.00 & 11.63 & 0.00 & \\
\hline 51 & & 223 & 32909.02 & 312129.4 & $\ldots$ & $\ldots$ & 15.66 & 0.06 & 12.63 & 0.01 & \\
\hline 157 & & & $\begin{array}{lll}3 & 29 & 09.20\end{array}$ & $\begin{array}{lll}31 & 27 & 15.5\end{array}$ & 17.07 & 0.03 & 16.15 & 0.02 & 15.66 & 0.04 & \\
\hline 57 & & 224 & $\begin{array}{lll}3 & 29 & 09.11\end{array}$ & $\begin{array}{lll}31 & 21 & 44.7\end{array}$ & $\ldots$ & $\ldots$ & 14.38 & 0.01 & 12.70 & 0.01 & \\
\hline 70 & & 225 & $\begin{array}{lll}3 & 29 & 09.27\end{array}$ & $\begin{array}{lll}31 & 21 & 04.3\end{array}$ & 16.07 & 0.02 & 14.24 & 0.01 & 13.14 & 0.01 & \\
\hline 60 & & & $\begin{array}{lll}3 & 29 & 09.44\end{array}$ & $\begin{array}{lll}31 & 27 & 20.5\end{array}$ & 13.94 & 0.00 & 13.18 & 0.00 & 12.84 & 0.01 & \\
\hline 7 & & 226 & $\begin{array}{lll}3 & 29 & 09.59\end{array}$ & $\begin{array}{lll}31 & 22 & 56.5\end{array}$ & 11.09 & 0.00 & 10.43 & 0.00 & 9.62 & 0.00 & SVS 7 \\
\hline 94 & & 228 & $\begin{array}{lll}3 & 29 & 10.15\end{array}$ & $\begin{array}{lll}31 & 27 & 15.4\end{array}$ & 15.30 & 0.01 & 14.63 & 0.01 & 14.24 & 0.01 & \\
\hline 164 & & & $\begin{array}{lll}3 & 29 & 10.34\end{array}$ & 312358.0 & 16.82 & 0.03 & 16.10 & 0.03 & 15.69 & 0.06 & \\
\hline 1 & & 230 & $\begin{array}{lll}3 & 29 & 10.32\end{array}$ & 312159.5 & $9.34^{\mathrm{c}}$ & $\ldots$ & $8.23^{\mathrm{c}}$ & $\ldots$ & $7.45^{\mathrm{c}}$ & $\ldots$ & SVS 3 , neb. \\
\hline 120 & & & $\begin{array}{lll}3 & 29 & 10.34\end{array}$ & 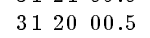 & 16.13 & 0.02 & 15.76 & 0.02 & 14.92 & 0.03 & \\
\hline 55 & & 231 & $\begin{array}{lll}3 & 29 & 10.41\end{array}$ & $\begin{array}{lll}31 & 23 & 34.9\end{array}$ & 15.51 & 0.01 & 13.75 & 0.00 & 12.68 & 0.01 & \\
\hline 121 & & 232 & $\begin{array}{lll}32 & 29 & 10.57\end{array}$ & $\begin{array}{lll}31 & 23 & 44.2\end{array}$ & 17.72 & 0.07 & 16.01 & 0.03 & 14.93 & 0.03 & \\
\hline 62 & & 233 & $\begin{array}{lll}32 & 29 & 10.75\end{array}$ & $\begin{array}{lll}31 & 22 & 30.2\end{array}$ & 14.56 & 0.01 & 13.48 & 0.01 & 12.92 & 0.01 & \\
\hline 162 & & & $\begin{array}{lll}3 & 29 & 10.82\end{array}$ & $\begin{array}{lll}31 & 19 & 53.6\end{array}$ & $\ldots$ & $\ldots$ & 18.00 & 0.22 & 15.68 & 0.07 & \\
\hline 49 & & 239 & $\begin{array}{lll}3 & 29 & 11.27\end{array}$ & $\begin{array}{lll}31 & 22 & 57.1\end{array}$ & 14.68 & 0.01 & 13.32 & 0.00 & 12.51 & 0.01 & \\
\hline 171 & 33 & & $\begin{array}{lll}3 & 29 & 11.50\end{array}$ & $\begin{array}{lll}31 & 18 & 28.4\end{array}$ & 18.38 & 0.13 & 17.79 & 0.19 & 15.81 & 0.13 & neb. in $\mathrm{HH} 6$ complex \\
\hline 118 & & 246 & 32911.71 & 312609.1 & 16.98 & 0.03 & 15.77 & 0.02 & 14.89 & 0.03 & \\
\hline 58 & 85 & 245 & $\begin{array}{lll}3 & 29 & 11.60\end{array}$ & $\begin{array}{lll}31 & 20 & 37.8\end{array}$ & 15.15 & 0.01 & 13.62 & 0.00 & 12.76 & 0.01 & \\
\hline 81 & & 244 & $\begin{array}{lll}32 & 29 & 11.78\end{array}$ & 312156.1 & 16.27 & 0.10 & 14.99 & 0.04 & 13.60 & 0.05 & \\
\hline 52 & & 248 & 32911.81 & 312127.4 & $\ldots$ & $\ldots$ & 15.32 & 0.03 & 12.66 & 0.01 & \\
\hline 124 & & & $\begin{array}{lll}3 & 29 & 11.98\end{array}$ & $\begin{array}{lll}31 & 25 & 48.7\end{array}$ & 15.82 & 0.01 & 15.33 & 0.01 & 15.01 & 0.03 & \\
\hline 161 & 31 & & $\begin{array}{lll}3 & 29 & 12.07\end{array}$ & $\begin{array}{lll}31 & 18 & 32.4\end{array}$ & $\ldots$ & $\ldots$ & 18.40 & 0.32 & 15.67 & 0.10 & neb. in $\mathrm{HH} 6$ complex \\
\hline 119 & & & $\begin{array}{lll}3 & 29 & 12.19\end{array}$ & $\begin{array}{lll}31 & 23 & 07.1\end{array}$ & 18.14 & 0.09 & 16.36 & 0.04 & 14.92 & 0.03 & \\
\hline 126 & & 251 & $\begin{array}{lll}3 & 29 & 12.31\end{array}$ & $\begin{array}{lll}31 & 27 & 22.7\end{array}$ & 15.55 & 0.01 & 14.99 & 0.01 & 15.03 & 0.03 & \\
\hline 75 & 83 & 257 & $\begin{array}{lll}3 & 29 & 12.73\end{array}$ & $\begin{array}{lll}31 & 20 & 07.9\end{array}$ & 14.47 & 0.01 & 13.78 & 0.00 & 13.33 & 0.01 & \\
\hline 42 & & 258 & $\begin{array}{lll}3 & 29 & 12.84\end{array}$ & $\begin{array}{lll}31 & 23 & 29.4\end{array}$ & 13.32 & 0.00 & 12.62 & 0.00 & 12.01 & 0.00 & \\
\hline 98 & 60 & 260 & $\begin{array}{lll}3 & 29 & 12.85\end{array}$ & 311845.6 & 17.21 & 0.04 & 16.89 & 0.07 & 14.30 & 0.02 & star in HH 6 complex \\
\hline 8 & & 262 & $329 \quad 13.05$ & $\begin{array}{lll}31 & 22 & 52.9\end{array}$ & 13.00 & 0.00 & 11.05 & 0.00 & 9.92 & 0.00 & \\
\hline 168 & 84 & & $\begin{array}{lll}3 & 29 & 13.10\end{array}$ & $\begin{array}{lll}31 & 19 & 49.9\end{array}$ & $\ldots$ & $\ldots$ & 17.41 & 0.11 & 15.75 & 0.06 & \\
\hline 184 & & & $\begin{array}{lll}3 & 29 & 13.43\end{array}$ & 312440.1 & 18.96 & 0.16 & 17.44 & 0.08 & 15.97 & 0.06 & \\
\hline 127 & & & $\begin{array}{lll}3 & 29 & 13.48\end{array}$ & $\begin{array}{lll}31 & 23 & 46.8\end{array}$ & 17.95 & 0.09 & 16.29 & 0.04 & 15.05 & 0.03 & \\
\hline 114 & & 266 & $\begin{array}{lll}329 & 14.03\end{array}$ & 312941.4 & 17.43 & 0.06 & 15.81 & 0.03 & 14.84 & 0.03 & \\
\hline 128 & 82 & & 32914.03 & $\begin{array}{lll}31 & 20 & 33.3\end{array}$ & 15.86 & 0.01 & 15.50 & 0.01 & 15.07 & 0.03 & \\
\hline 187 & & & $\begin{array}{lll}329 & 14.15\end{array}$ & 312247.8 & $\ldots$ & $\ldots$ & $\ldots$ & $\ldots$ & 16.00 & 0.08 & HH 344 neb. \\
\hline 66 & & 269 & $\begin{array}{lll}3 & 29 & 14.36\end{array}$ & $\begin{array}{lll}31 & 22 & 36.2\end{array}$ & 14.34 & 0.00 & 13.55 & 0.00 & 13.00 & 0.01 & \\
\hline 214 & & & $\begin{array}{lll}3 & 29 & 14.87\end{array}$ & $\begin{array}{lll}31 & 23 & 25.1\end{array}$ & 18.57 & 0.11 & 17.24 & 0.07 & 16.43 & 0.08 & \\
\hline 158 & & & $\begin{array}{lll}3 & 29 & 15.30\end{array}$ & $\begin{array}{lll}31 & 29 & 34.1\end{array}$ & $\ldots$ & $\ldots$ & 18.12 & 0.18 & 15.66 & 0.05 & \\
\hline
\end{tabular}


TABLE $1-$ Continued

\begin{tabular}{|c|c|c|c|c|c|c|c|c|c|c|c|}
\hline $\mathrm{MBO}^{\mathrm{a}}$ & $\mathrm{ASR}^{\mathrm{b}}$ & $\mathrm{LAL}^{\mathrm{c}}$ & $\mathrm{RA}(2000)$ & $\mathrm{DEC}(2000)$ & $\mathrm{J}^{\mathrm{d}}$ & $\sigma_{J}^{\mathrm{e}}$ & $\mathrm{H}$ & $\sigma_{H}$ & $\mathrm{~K}$ & $\sigma_{K}$ & Comments \\
\hline 147 & & & 32915.54 & $\begin{array}{lll}31 & 22 & 43.1\end{array}$ & 18.13 & 0.10 & 16.81 & 0.06 & 15.51 & 0.06 & \\
\hline 218 & 59 & & $\begin{array}{lll}3 & 29 & 15.58\end{array}$ & $\begin{array}{lll}31 & 19 & 11.5\end{array}$ & $\ldots$ & $\ldots$ & 19.17 & 0.43 & 16.51 & 0.11 & \\
\hline 131 & & 277 & 32916.50 & 312446.0 & 16.77 & 0.03 & 15.83 & 0.02 & 15.17 & 0.03 & \\
\hline 24 & & 276 & $\begin{array}{lll}3 & 29 & 16.53\end{array}$ & $\begin{array}{lll}31 & 23 & 49.6\end{array}$ & 13.18 & 0.00 & 11.84 & 0.00 & 11.08 & 0.00 & \\
\hline 48 & & 278 & $\begin{array}{lll}3 & 29 & 16.49\end{array}$ & $\begin{array}{lll}31 & 21 & 02.8\end{array}$ & 14.83 & 0.01 & 13.26 & 0.00 & 12.43 & 0.01 & \\
\hline 79 & & 279 & $\begin{array}{lll}3 & 29 & 16.75\end{array}$ & $\begin{array}{lll}31 & 23 & 25.3\end{array}$ & 15.18 & 0.01 & 14.30 & 0.01 & 13.54 & 0.01 & \\
\hline 136 & & & $\begin{array}{lll}3 & 29 & 17.43\end{array}$ & 312454.6 & $\ldots$ & $\ldots$ & 17.07 & 0.09 & 15.22 & 0.06 & \\
\hline 195 & & & $\begin{array}{lll}3 & 29 & 17.58\end{array}$ & $\begin{array}{lll}31 & 24 & 43.8\end{array}$ & 17.11 & 0.05 & 16.58 & 0.06 & 16.16 & 0.11 & \\
\hline 2 & & 283 & $\begin{array}{lll}3 & 29 & 17.60\end{array}$ & $\begin{array}{lll}31 & 22 & 45.1\end{array}$ & $9.78^{\mathrm{c}}$ & $\ldots$ & $8.93^{\mathrm{c}}$ & $\ldots$ & $8.32^{\mathrm{c}}$ & $\ldots$ & LkH $\alpha 270$, SVS 2, neb. \\
\hline 185 & & & $\begin{array}{lll}3 & 29 & 17.61\end{array}$ & 312046.0 & 17.04 & 0.03 & 16.31 & 0.03 & 15.97 & 0.06 & \\
\hline 64 & 80 & 286 & $\begin{array}{lll}3 & 29 & 17.70\end{array}$ & $\begin{array}{lll}31 & 19 & 48.5\end{array}$ & 14.60 & 0.01 & 13.57 & 0.00 & 12.98 & 0.01 & \\
\hline 16 & & 293 & $\begin{array}{lll}3 & 29 & 18.67\end{array}$ & $\begin{array}{lll}31 & 23 & 25.5\end{array}$ & 11.25 & 0.00 & 10.72 & 0.00 & 10.28 & 0.00 & \\
\hline 109 & 81 & & $\begin{array}{lll}3 & 29 & 18.61\end{array}$ & $\begin{array}{lll}31 & 20 & 18.1\end{array}$ & 18.31 & 0.09 & 16.22 & 0.03 & 14.70 & 0.02 & $4^{\prime \prime}$ from MBO 154 \\
\hline 154 & & & 32918.61 & $\begin{array}{lll}31 & 20 & 21.7\end{array}$ & 18.89 & 0.15 & 17.04 & 0.05 & 15.61 & 0.05 & $4^{\prime \prime}$ from MBO 109 \\
\hline 4 & & 295 & $\begin{array}{lll}3 & 29 & 19.72\end{array}$ & 312456.9 & $8.81^{\mathrm{C}}$ & $\ldots$ & $8.59^{c}$ & $\ldots$ & $8.46^{\mathrm{C}}$ & $\ldots$ & $\mathrm{BD}+30549$, SVS 1, neb. \\
\hline 203 & & & $\begin{array}{lll}3 & 29 & 19.72\end{array}$ & 312258.0 & 15.99 & 0.02 & 16.12 & 0.04 & 16.24 & 0.10 & in neb. from $\mathrm{LkH} \alpha 270$ \\
\hline 76 & 77 & 297 & $\begin{array}{lll}3 & 29 & 19.83\end{array}$ & $\begin{array}{lll}31 & 18 & 48.1\end{array}$ & 17.21 & 0.04 & 14.67 & 0.01 & 13.35 & 0.01 & \\
\hline 46 & & 296 & $\begin{array}{lll}3 & 29 & 19.98\end{array}$ & 312407.6 & 18.48 & 0.16 & 14.93 & 0.01 & 12.16 & 0.01 & \\
\hline 115 & & 299 & 32920.49 & 312634.3 & 16.56 & 0.02 & 15.26 & 0.01 & 14.87 & 0.02 & \\
\hline 17 & 112 & 300 & $\begin{array}{lll}3 & 29 & 20.38\end{array}$ & $\begin{array}{llll}31 & 18 & 34.4\end{array}$ & 14.25 & 0.01 & 12.14 & 0.00 & 10.59 & 0.00 & SVS 5,star in $\mathrm{HH} 17$ neb. \\
\hline 174 & & & 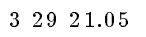 & 312159.7 & 17.43 & 0.05 & 16.38 & 0.03 & 15.85 & 0.06 & \\
\hline 61 & & 302 & $\begin{array}{lll}3 & 29 & 21.22\end{array}$ & $\begin{array}{lll}31 & 23 & 46.4\end{array}$ & 17.43 & 0.05 & 14.66 & 0.01 & 12.91 & 0.01 & \\
\hline 183 & & & $\begin{array}{lll}3 & 29 & 21.32\end{array}$ & 312254.5 & $\ldots$ & $\ldots$ & 17.46 & 0.10 & 15.96 & 0.07 & \\
\hline 41 & & 303 & $\begin{array}{lll}3 & 29 & 21.44\end{array}$ & $\begin{array}{lll}31 & 25 & 12.6\end{array}$ & 13.14 & 0.00 & 12.43 & 0.00 & 12.00 & 0.01 & \\
\hline 32 & & 304 & $\begin{array}{lll}3 & 29 & 21.49\end{array}$ & $\begin{array}{lll}31 & 21 & 10.7\end{array}$ & 12.25 & 0.00 & 11.84 & 0.00 & 11.45 & 0.00 & \\
\hline 23 & & 306 & 32921.96 & $\begin{array}{lll}31 & 24 & 15.4\end{array}$ & 11.89 & 0.00 & 11.41 & 0.00 & 11.06 & 0.00 & \\
\hline 186 & & & $\begin{array}{lll}329 & 21.95\end{array}$ & $\begin{array}{lll}31 & 20 & 44.5\end{array}$ & 17.05 & 0.03 & 16.18 & 0.03 & 15.98 & 0.07 & \\
\hline 177 & & & $\begin{array}{lll}329 & 22.30\end{array}$ & $\begin{array}{lll}31 & 21 & 37.1\end{array}$ & 17.61 & 0.05 & 16.53 & 0.04 & 15.91 & 0.05 & \\
\hline 167 & & & $\begin{array}{lll}3 & 29 & 22.87\end{array}$ & $\begin{array}{lll}31 & 22 & 35.4\end{array}$ & 16.82 & 0.02 & 16.15 & 0.02 & 15.74 & 0.05 & \\
\hline 207 & & & $\begin{array}{lll}3 & 29 & 22.96\end{array}$ & $\begin{array}{lll}31 & 26 & 31.1\end{array}$ & 19.16 & 0.20 & 17.75 & 0.09 & 16.28 & 0.08 & \\
\hline 104 & & 309 & $\begin{array}{lll}3 & 29 & 23.21\end{array}$ & 312953.6 & 15.97 & 0.02 & 15.01 & 0.01 & 14.61 & 0.03 & \\
\hline 201 & & & $\begin{array}{lll}3 & 29 & 23.11\end{array}$ & $\begin{array}{lll}31 & 23 & 57.3\end{array}$ & $\ldots$ & $\ldots$ & 17.76 & 0.11 & 16.21 & 0.08 & \\
\hline 54 & & 308 & $\begin{array}{lll}3 & 29 & 23.18\end{array}$ & $\begin{array}{lll}31 & 26 & 52.9\end{array}$ & 13.67 & 0.00 & 12.96 & 0.00 & 12.67 & 0.01 & \\
\hline 30 & 78 & 310 & $\begin{array}{lll}3 & 29 & 23.08\end{array}$ & $\begin{array}{lll}31 & 20 & 30.6\end{array}$ & 12.26 & 0.00 & 11.73 & 0.00 & 11.37 & 0.00 & \\
\hline 166 & & & $\begin{array}{lll}3 & 29 & 23.19\end{array}$ & 312446.5 & 19.08 & 0.23 & 17.14 & 0.07 & 15.70 & 0.07 & \\
\hline 204 & & & $\begin{array}{lll}3 & 29 & 23.23\end{array}$ & $\begin{array}{lll}31 & 25 & 43.4\end{array}$ & $\ldots$ & $\ldots$ & 20.07 & 1.20 & 16.24 & 0.07 & \\
\hline 29 & & 311 & $\begin{array}{lll}329 & 23.41\end{array}$ & 312331.0 & 12.65 & 0.00 & 11.87 & 0.00 & 11.32 & 0.00 & \\
\hline 56 & & 312 & $\begin{array}{lll}3 & 29 & 23.62\end{array}$ & $\begin{array}{lll}31 & 25 & 09.3\end{array}$ & 13.91 & 0.00 & 13.11 & 0.00 & 12.69 & 0.01 & \\
\hline 193 & & & $\begin{array}{lll}3 & 29 & 24.18\end{array}$ & $\begin{array}{lll}31 & 29 & 33.5\end{array}$ & 17.77 & 0.08 & 16.53 & 0.04 & 16.14 & 0.08 & \\
\hline 85 & 79 & 313 & $\begin{array}{lll}3 & 29 & 24.02\end{array}$ & $\begin{array}{lll}31 & 19 & 57.9\end{array}$ & 15.45 & 0.01 & 14.22 & 0.01 & 13.74 & 0.01 & star in $\mathrm{HH} 4$ neb. \\
\hline 69 & & 314 & $\begin{array}{lll}3 & 29 & 24.41\end{array}$ & $\begin{array}{lll}31 & 28 & 14.5\end{array}$ & 14.28 & 0.00 & 13.38 & 0.00 & 13.12 & 0.01 & \\
\hline 77 & & 315 & $\begin{array}{lll}329 & 24.76\end{array}$ & $\begin{array}{lll}31 & 24 & 06.3\end{array}$ & 14.27 & 0.00 & 13.81 & 0.00 & 13.36 & 0.01 & \\
\hline 129 & & & 32925.25 & 312150.1 & 16.74 & 0.03 & 15.66 & 0.02 & 15.09 & 0.03 & \\
\hline 9 & & 318 & $\begin{array}{lll}329 & 25.87\end{array}$ & $\begin{array}{lll}31 & 26 & 39.9\end{array}$ & 11.00 & 0.00 & 10.56 & 0.00 & 10.01 & 0.00 & SVS 4, double \\
\hline 176 & & & 32926.01 & $\begin{array}{lll}31 & 23 & 44.9\end{array}$ & 17.66 & 0.06 & 16.42 & 0.03 & 15.91 & 0.06 & \\
\hline 137 & & & $\begin{array}{lll}3 & 29 & 25.97\end{array}$ & 312055.9 & 16.79 & 0.02 & 15.78 & 0.02 & 15.27 & 0.03 & \\
\hline 153 & & & $\begin{array}{lll}3 & 29 & 26.26\end{array}$ & $\begin{array}{lll}31 & 22 & 50.3\end{array}$ & 17.30 & 0.03 & 16.14 & 0.02 & 15.60 & 0.04 & \\
\hline 15 & & 321 & $\begin{array}{lll}3 & 29 & 26.76\end{array}$ & $\begin{array}{lll}31 & 26 & 47.2\end{array}$ & 10.97 & 0.00 & 10.52 & 0.00 & 10.27 & 0.00 & SVS 6 \\
\hline 202 & & & $\begin{array}{lll}3 & 29 & 27.24\end{array}$ & $\begin{array}{lll}31 & 29 & 32.0\end{array}$ & 18.26 & 0.12 & 17.16 & 0.08 & 16.23 & 0.08 & \\
\hline 72 & & 324 & $\begin{array}{lll}3 & 29 & 27.54\end{array}$ & $\begin{array}{lll}31 & 21 & 10.3\end{array}$ & 14.64 & 0.01 & 13.57 & 0.00 & 13.23 & 0.01 & \\
\hline 111 & & 325 & $\begin{array}{lll}3 & 29 & 27.78\end{array}$ & $\begin{array}{lll}31 & 21 & 20.3\end{array}$ & 16.17 & 0.02 & 15.09 & 0.01 & 14.73 & 0.02 & $3.5^{\prime \prime}$ from $\mathrm{MBO} 110$ \\
\hline 133 & & & 32927.94 & $\begin{array}{lll}31 & 25 & 10.6\end{array}$ & 17.54 & 0.04 & 16.08 & 0.02 & 15.18 & 0.03 & \\
\hline 212 & & & 32928.03 & 312235.6 & 18.01 & 0.07 & 16.88 & 0.05 & 16.35 & 0.09 & \\
\hline 110 & & & 32928.01 & 312118.0 & 16.02 & 0.01 & 15.01 & 0.01 & 14.72 & 0.02 & $3.5^{\prime \prime}$ from $\mathrm{MBO} 111$ \\
\hline 101 & & 326 & $\begin{array}{lll}3 & 29 & 28.10\end{array}$ & $\begin{array}{lll}31 & 23 & 59.2\end{array}$ & 16.30 & 0.02 & 15.01 & 0.01 & 14.41 & 0.02 & \\
\hline 59 & & 327 & 32928.01 & $\begin{array}{lll}31 & 18 & 38.9\end{array}$ & 15.01 & 0.01 & 13.54 & 0.00 & 12.82 & 0.01 & \\
\hline 181 & & & $\begin{array}{lll}3 & 29 & 28.93\end{array}$ & 312428.8 & 17.33 & 0.04 & 16.24 & 0.03 & 15.93 & 0.05 & \\
\hline 210 & & & $\begin{array}{lll}3 & 29 & 29.39\end{array}$ & $\begin{array}{lll}31 & 27 & 03.6\end{array}$ & 17.16 & 0.03 & 16.47 & 0.03 & 16.30 & 0.08 & \\
\hline 22 & & 331 & $\begin{array}{lll}3 & 29 & 29.22\end{array}$ & $31 \quad 18 \quad 34.5$ & 12.51 & 0.00 & 11.36 & 0.00 & 11.05 & 0.00 & \\
\hline 222 & & & $\begin{array}{lll}3 & 29 & 29.50\end{array}$ & $\begin{array}{lll}31 & 23 & 59.7\end{array}$ & 18.11 & 0.09 & 17.35 & 0.07 & 16.94 & 0.15 & \\
\hline 27 & & 333 & $\begin{array}{lll}3 & 29 & 29.72\end{array}$ & $\begin{array}{lll}31 & 21 & 02.9\end{array}$ & 12.49 & 0.00 & 11.60 & 0.00 & 11.32 & 0.00 & \\
\hline 68 & & 335 & $\begin{array}{lll}3 & 29 & 30.49\end{array}$ & $\begin{array}{lll}31 & 27 & 27.9\end{array}$ & 13.91 & 0.00 & 13.42 & 0.00 & 13.11 & 0.01 & \\
\hline 28 & & 336 & $\begin{array}{lll}3 & 29 & 30.34\end{array}$ & $\begin{array}{lll}31 & 19 & 03.5\end{array}$ & 11.98 & 0.00 & 11.30 & 0.00 & 11.32 & 0.00 & \\
\hline 135 & & & $\begin{array}{lll}3 & 29 & 30.53\end{array}$ & $\begin{array}{lll}31 & 23 & 11.5\end{array}$ & 17.54 & 0.04 & 16.04 & 0.02 & 15.21 & 0.04 & \\
\hline
\end{tabular}


TABle 1-Continued

\begin{tabular}{|c|c|c|c|c|c|c|c|c|c|c|c|}
\hline $\mathrm{MBO}^{\mathrm{a}}$ & $\mathrm{ASR}^{\mathrm{b}}$ & $\mathrm{LAL}^{\mathrm{c}}$ & $\mathrm{RA}(2000)$ & $\operatorname{DEC}(2000)$ & $\mathrm{J}^{\mathrm{d}}$ & $\sigma_{J}^{\mathrm{e}}$ & $\mathrm{H}$ & $\sigma_{H}$ & $\mathrm{~K}$ & $\sigma_{K}$ & Comments \\
\hline 25 & & 337 & 32930.76 & $\begin{array}{lll}31 & 23 & 52.9\end{array}$ & 12.75 & 0.00 & 11.71 & 0.00 & 11.21 & 0.00 & \\
\hline 163 & & & $\begin{array}{lll}32 & 29 & 31.46\end{array}$ & $\begin{array}{lll}31 & 25 & 27.4\end{array}$ & 17.02 & 0.03 & 16.49 & 0.04 & 15.68 & 0.05 & \\
\hline 40 & & 342 & $\begin{array}{lll}3 & 29 & 32.46\end{array}$ & 312436.8 & 12.88 & 0.00 & 12.71 & 0.00 & 12.00 & 0.00 & \\
\hline 53 & & 344 & $\begin{array}{lll}329 & 32.80\end{array}$ & $\begin{array}{lll}31 & 27 & 12.7\end{array}$ & 13.59 & 0.00 & 12.94 & 0.00 & 12.66 & 0.01 & \\
\hline 178 & & & $\begin{array}{lll}3 & 29 & 32.81\end{array}$ & 312205.5 & 17.22 & 0.04 & 16.37 & 0.03 & 15.92 & 0.07 & \\
\hline 190 & & & $\begin{array}{lll}3 & 29 & 33.44\end{array}$ & $\begin{array}{lll}31 & 20 & 42.3\end{array}$ & 17.29 & 0.04 & 16.29 & 0.03 & 16.07 & 0.07 & \\
\hline 140 & & & 32933.80 & 312036.2 & 16.24 & 0.02 & 15.74 & 0.02 & 15.38 & 0.04 & \\
\hline 196 & & & $\begin{array}{lll}3 & 29 & 34.12\end{array}$ & 312334.0 & 19.13 & 0.22 & 17.24 & 0.07 & 16.17 & 0.07 & \\
\hline 103 & & 346 & $\begin{array}{lll}3 & 29 & 34.22\end{array}$ & $\begin{array}{lll}31 & 23 & 42.7\end{array}$ & 16.61 & 0.03 & 15.24 & 0.01 & 14.52 & 0.02 & \\
\hline 160 & & & $\begin{array}{lll}3 & 29 & 34.22\end{array}$ & $\begin{array}{lll}31 & 21 & 55.1\end{array}$ & 16.74 & 0.03 & 15.97 & 0.02 & 15.67 & 0.05 & \\
\hline 211 & & & $\begin{array}{lll}329 & 34.36\end{array}$ & $\begin{array}{lll}31 & 28 & 29.9\end{array}$ & $\ldots$ & $\ldots$ & 17.63 & 0.15 & 16.31 & 0.13 & \\
\hline 74 & & 348 & $\begin{array}{lll}3 & 29 & 34.33\end{array}$ & $\begin{array}{llll}31 & 19 & 10.7\end{array}$ & 14.51 & 0.01 & 13.27 & 0.00 & 13.32 & 0.01 & \\
\hline 43 & & 350 & 32934.69 & $\begin{array}{lll}31 & 29 & 07.8\end{array}$ & 13.85 & 0.00 & 12.38 & 0.00 & 12.02 & 0.01 & \\
\hline 200 & & & $\begin{array}{lll}32 & 29 & 35.22\end{array}$ & $31 \quad 1911.0$ & 18.22 & 0.15 & 16.98 & 0.08 & 16.20 & 0.10 & \\
\hline 189 & & & $\begin{array}{lll}3 & 29 & 36.29\end{array}$ & 311945.4 & 17.27 & 0.05 & 16.20 & 0.04 & 16.06 & 0.08 & \\
\hline 10 & & 355 & 32936.51 & 312946.5 & $\ldots$ & $\ldots$ & 10.59 & 0.01 & 10.04: & 0.00 & edge of $K$ frame \\
\hline 99 & & 356 & $\begin{array}{lll}329 & 36.73\end{array}$ & $\begin{array}{lll}31 & 28 & 46.7\end{array}$ & 14.76 & 0.01 & 14.24: & 0.01 & 14.30 & 0.02 & edge of $\mathrm{H}$ frame \\
\hline 159 & & & 32936.81 & $\begin{array}{lll}31 & 26 & 39.4\end{array}$ & 17.33 & 0.05 & 15.99 & 0.03 & 15.67 & 0.05 & \\
\hline 65 & & & $\begin{array}{lll}329 & 37.65\end{array}$ & $\begin{array}{lll}31 & 22 & 02.8\end{array}$ & 13.72 & 0.00 & 13.23 & 0.00 & 13.00 & 0.01 & \\
\hline 175 & & & 32937.61 & $\begin{array}{lll}31 & 18 & 43.0\end{array}$ & 17.24 & 0.05 & 16.13 & 0.04 & 15.88 & 0.08 & \\
\hline
\end{tabular}

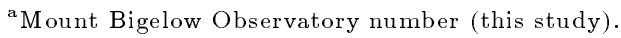

${ }^{\mathrm{b}}$ Aspin, Sandell, \& Russell 1994

${ }^{\mathrm{c}}$ Lada et al. 1996

${ }^{\mathrm{d}} \mathrm{JHK}$ photometry transformed into the CIT photometric system (see text).

e " $\sigma$ " values quoted for the JHK photometry are the statistical errors only and can be exceeded by uncorrected variations in the pixel to pixel response across the array of $\pm 0.05 \mathrm{mag}$ in the central regions of the mosaic and as large as $\pm 0.10 \mathrm{mag}$ in the outer regions. 
TABLE 2. Log of Brown Dwarf Candidate Observations

\begin{tabular}{|c|c|c|c|c|c|c|}
\hline $\begin{array}{l}\text { Object }^{\mathrm{a}} \\
3^{h},+31^{\circ}\end{array}$ & MBO No.b & ASR No. ${ }^{c}$ & LAL No.d & X-Ray ID & $\begin{array}{l}\text { Int. Time } \\
(\mathrm{sec})\end{array}$ & $\mathrm{SNR}_{\text {cont }}{ }^{\mathrm{f}}$ \\
\hline $2852.7+1626$ & $\ldots$ & 46 & 128 & $\ldots$ & 600 & 85 \\
\hline $2853.9+1654$ & $\cdots$ & 42 & 131 & Ch26 & 960 & 120 \\
\hline $2855.2+1735$ & $\ldots$ & 38 & 142 & $\ldots$ & 960 & 55 \\
\hline $2856.8+1550$ & $\ldots$ & 15 & 153 & $\cdots$ & 1200 & 40 \\
\hline $2856.9+2049$ & 91 & $\cdots$ & 152 & $\cdots$ & 2160 & 35 \\
\hline $2857.0+1534$ & $\cdots$ & 17 & 156 & $\ldots$ & 1320 & 65 \\
\hline $2858.2+2209$ & 73 & $\cdots$ & 164 & tL164 & 960 & 60 \\
\hline $2858.4+2257$ & 80 & $\cdots$ & 165 & tL165 & 1200 & 50 \\
\hline $2900.2+1339$ & $\ldots$ & 51 & 178 & R12,Ch43 & 1440 & 55 \\
\hline $2900.3+2046$ & 45 & 133 & 177 & Ch44 & 720 & 95 \\
\hline $2902.0+1611$ & $\cdots$ & 3 & 184 & Ch47 & 1200 & 55 \\
\hline $2902.8+1601$ & $\cdots$ & 2 & 188 & R13,Ch48 & 480 & 75 \\
\hline $2903.3+1840$ & 88 & 63 & 193 & $\cdots$ & 1440 & 40 \\
\hline $2910.8+2230$ & 62 & $\ldots$ & 233 & Ch66 & 960 & 55 \\
\hline $2911.2+1717$ & $\cdots$ & 24 & 243 & Ch67 & 720 & 75 \\
\hline $2912.7+2008$ & 75 & 83 & 257 & Ch70 & 1200 & 40 \\
\hline $2914.1+2236$ & 66 & $\cdots$ & 269 & Ch75 & 720 & 60 \\
\hline $2916.8+2325$ & 79 & $\cdots$ & 279 & $\cdots$ & 960 & 45 \\
\hline $2917.7+1948$ & 64 & 80 & 286 & $\ldots$ & 1200 & 70 \\
\hline $2923.2+2653$ & 54 & $\cdots$ & 308 & $\cdots$ & 720 & 105 \\
\hline $2923.6+2509$ & 56 & $\ldots$ & 312 & Ch94 & 960 & 70 \\
\hline $2924.0+1958$ & 85 & 79 & 313 & $\ldots$ & 1440 & 50 \\
\hline $2924.4+2814$ & 69 & $\ldots$ & 314 & tL314 & 1080 & 70 \\
\hline $2924.8+2406$ & 77 & $\ldots$ & 315 & Ch97 & 960 & 75 \\
\hline $2932.8+2713$ & 53 & $\ldots$ & 344 & Ch106 & 720 & 80 \\
\hline
\end{tabular}

${ }^{a}$ Object RA (min, sec) and DEC (', ") in J2000.0

bMount Bigelow Observatory number, this study

${ }^{\mathrm{c}}$ Aspin, Sandell,\& Russell 1994

${ }^{\mathrm{d}}$ Lada, Alves, \& Lada 1996

'X-ray source from the ROSAT study of Preibisch (R, 1997) or the CHANDRA study of Getman et al. (Ch, 2002). "tL" denotes a tentative x-ray detection of a LAL source by Getman et al.

${ }^{f}$ The signal-to-noise ratio measured from the continuum of a normalized spectrum. 
TABle 3. NGC 1333 Brown Dwarf Candidate Properties

\begin{tabular}{|c|c|c|c|c|c|c|c|c|c|c|c|}
\hline Object & $\mathrm{Q}^{\mathrm{a}}$ & Sp. Type & $\log \left(\mathrm{T}_{e f f}\right)$ & $(\mathrm{J}-\mathrm{H})$ & $(\mathrm{H}-\mathrm{K})$ & $\mathrm{K}$ & $\mathrm{A}_{v}^{\mathrm{b}}$ & $\log \left(\mathrm{L}_{b o l} / \mathrm{L}_{\odot}\right)$ & $r_{k}^{\mathrm{c}}$ & Phot? ${ }^{\mathrm{d}}$ & Comments \\
\hline ASR 46 & 0.61 & M6.2 & 3.436 & 0.60 & 0.43 & 12.56 & 0 & -1.44 & 0.08 & LAL & bd? \\
\hline ASR 42 & 0.60 & M6.3 & 3.433 & 0.85 & 0.46 & 11.73 & 2 & -0.98 & -0.04 & LAL & $\ldots$ \\
\hline ASR 38 & 0.52 & M7.9 & 3.389 & 0.71 & 0.70 & 13.73 & 1 & -1.98 & 0.22 & LAL & bd \\
\hline ASR 15 & 0.54 & M7.4 & 3.403 & 0.81 & 0.58 & 13.61 & 2 & -1.82 & 0.06 & LAL & $\mathrm{bd}$ \\
\hline MBO 91 & 0.57 & M6.8 & 3.420 & 0.82 & 0.51 & 13.99 & 2 & -1.93 & 0.02 & LAL & $\mathrm{bd}$ \\
\hline ASR 17 & 0.54 & M7.4 & 3.403 & 1.25 & 0.74 & 13.23 & 6 & -1.49 & -0.04 & LAL & $\mathrm{bd}$ \\
\hline MBO 73 & 0.60 & $\mathrm{M} 6.4$ & 3.431 & 1.43 & 0.87 & 13.40 & 7 & -1.48 & 0.02 & LAL & bd? \\
\hline MBO 80 & 0.56 & M7.1 & 3.412 & 0.94 & 0.72 & 13.57 & 3 & -1.79 & 0.13 & $\mathrm{MBO}$ & $\mathrm{bd}$ \\
\hline ASR 51 & 0.78 & $\mathrm{M} 2.7$ & 3.520 & 1.04 & 0.52 & 11.51 & 4 & -0.66 & 0.03 & LAL & $\ldots$ \\
\hline MBO 45 & 0.59 & M6.6 & 3.425 & 0.96 & 0.51 & 11.94 & 3 & -1.03 & -0.06 & LAL & bd? \\
\hline ASR 3 & 0.71 & $\mathrm{M} 4.2$ & 3.486 & 0.57 & 0.19 & 13.72 & 0 & -1.76 & -0.06 & LAL & $\ldots$ \\
\hline ASR 2 & 0.80 & M2.3 & 3.528 & 0.59 & 0.19 & 11.99 & 0 & -0.95 & -0.02 & LAL & $\ldots$ \\
\hline ASR 63 & 0.54 & M7.4 & 3.403 & 0.99 & 0.74 & 13.87 & 3 & -1.89 & 0.11 & $\mathrm{MBO}$ & $\mathrm{bd}$ \\
\hline MBO 62 & 0.55 & M7.4 & 3.403 & 1.05 & 0.60 & 12.91 & 4 & -1.42 & -0.06 & LAL & bd? \\
\hline ASR 24 & 0.51 & M8.2 & 3.380 & 0.67 & 0.41 & 12.84 & 0 & -1.54 & -0.04 & ASR & $\mathrm{bd}$ \\
\hline ASR 83 & 0.53 & M7.7 & 3.394 & 0.64 & 0.40 & 13.37 & 0 & -1.76 & -0.02 & LAL & $\mathrm{bd}$ \\
\hline MBO 66 & 0.58 & M6.6 & 3.425 & 0.79 & 0.55 & 13.00 & 2 & -1.57 & 0.07 & $\mathrm{MBO}$ & bd? \\
\hline MBO 79 & 0.79 & M2.5 & 3.524 & 0.88 & 0.76 & 13.54 & 3 & -1.66 & 0.39 & $\mathrm{MBO}$ & $\ldots$ \\
\hline ASR 80 & 0.59 & M6.5 & 3.428 & 0.99 & 0.54 & 13.08 & 3 & -1.48 & -0.05 & LAL & bd? \\
\hline MBO 54 & 0.64 & M5.6 & 3.452 & 0.72 & 0.38 & 12.32 & 1 & -1.24 & -0.02 & LAL & . \\
\hline MBO 56 & 0.64 & M5.5 & 3.454 & 1.00 & 0.42 & 12.45 & 4 & -1.15 & -0.13 & LAL & $\cdots$ \\
\hline ASR 79 & 0.78 & M2.6 & 3.522 & 0.98 & 0.27 & 13.83 & 4 & -1.52 & -0.16 & LAL & $\cdots$ \\
\hline MBO 69 & 0.55 & M7.4 & 3.403 & 0.78 & 0.35 & 12.84 & 1 & -1.44 & -0.13 & LAL & bd \\
\hline MBO 77 & 0.58 & M6.7 & 3.423 & 0.54 & 0.30 & 13.43 & $0^{\mathrm{f}}$ & -1.70 & -0.03 & LAL & bd? \\
\hline MBO 53 & 0.67 & M4.9 & 3.469 & 0.56 & 0.39 & 12.24 & 0 & -1.27 & 0.09 & LAL & $\ldots$ \\
\hline
\end{tabular}

${ }^{\mathrm{a}}$ Water vapor index Q. One sigma errors range from $0.010-0.022$ with a median error of 0.014 .

${ }^{\mathrm{b}}$ Visual extinction $\mathrm{A}_{v}=9.09 \times\left[(J-H)_{o b s}-(J-H)_{0}\right]$.

${ }^{c}$ Excess emission at $\lambda=2.2 \mu \mathrm{m}, r_{k}=F_{K e x} / F_{K *}$, assuming no excess emission at H. Estimates are lower limits to the true values.

${ }^{\mathrm{d}}$ Infrared photometry obtained by this group (MBO), the study of Aspin et al. (ASR, 1994), or that of Lada et al. (LAL, 1996) and transformed into the CIT system (see text).

eObjects that lie $250 \mathrm{~K}$ or more to the right of the hydrogen burning limit shown in Fig. 6a are denoted by "bd" and objects that lie in the brown dwarf regime within $250 \mathrm{~K}$ of the hydrogen burning limit are denoted by "bd?".

${ }^{f}$ Observed $(J-H)$ colors are smaller than instrinsic color implied by spectral type. The luminosity was estimated assuming the visual extinction was zero. 